\title{
Statistical analyses of Higgs- and $Z$-portal dark matter models
}

\author{
John Ellis, ${ }^{1,2,3}$ Andrew Fowlie, ${ }^{4}$ Luca Marzola, ${ }^{2}$ and Martti Raidal ${ }^{2,3}$ \\ ${ }^{1}$ Theoretical Particle Physics and Cosmology Group, Department of Physics, \\ King's College London, London WC2R 2LS, United Kingdom \\ ${ }^{2}$ National Institute of Chemical Physics and Biophysics, Rävala 10, Tallinn 10143, Estonia \\ ${ }^{3}$ Theoretical Physics Department, CERN, Geneva 23 CH-1211, Switzerland \\ ${ }^{4}$ ARC Centre of Excellence for Particle Physics at the Tera-scale, Monash University, Melbourne, \\ Victoria 3800, Australia
}

(Received 12 March 2018; published 11 June 2018)

\begin{abstract}
We perform frequentist and Bayesian statistical analyses of Higgs- and Z-portal models of dark matter particles with spin $0,1 / 2$, and 1 . Our analyses incorporate data from direct detection and indirect detection experiments, as well as LHC searches for monojet and monophoton events, and we also analyze the potential impacts of future direct detection experiments. We find acceptable regions of the parameter spaces for Higgsportal models with real scalar, neutral vector, Majorana, or Dirac fermion dark matter particles, and Z-portal models with Majorana or Dirac fermion dark matter particles. In many of these cases, there are interesting prospects for discovering dark matter particles in Higgs or $Z$ decays, as well as dark matter particles weighing $\gtrsim 100 \mathrm{GeV}$. Negative results from planned direct detection experiments would still allow acceptable regions for Higgs- and Z-portal models with Majorana or Dirac fermion dark matter particles.
\end{abstract}

DOI: 10.1103/PhysRevD.97.115014

\section{INTRODUCTION}

In the last few decades, numerous astrophysical and cosmological observations have confirmed the need for dark matter (DM) via its gravitational interactions, with the most precise estimate of its density being provided by Planck satellite measurements of the cosmic microwave background radiation in combination with other experiments [1]. However, many experimental searches have failed to find conclusive evidence for DM via nongravitational interactions. This year alone, world-leading upper limits on the strength of DM interactions with matter were set by the PICO [2], XENON1T [3], and PandaX [4] direct detection (DD) experiments, as well as by monojet [5-9] and monophoton $[10,11]$ searches for DM at the LHC. Thus, to date, the experimental evidence for DM remains limited to its gravitational interactions.

In many models, DM is composed of weakly interacting massive particles (WIMPs) that were in thermal equilibrium with Standard Model (SM) particles in the early Universe. In such a case, the DM relic abundance is predicted to be that at which WIMPs freeze out from thermal equilibrium in the early Universe. WIMP decays are typically forbidden by a symmetry, e.g., by $\mathbb{Z}_{2,3}$

Published by the American Physical Society under the terms of the Creative Commons Attribution 4.0 International license. Further distribution of this work must maintain attribution to the author(s) and the published article's title, journal citation, and DOI. Funded by SCOAP ${ }^{3}$.
[12-14] or more generally by $\mathbb{Z}_{N}[15,16]$, and the predicted WIMP abundance agrees "miraculously" with the astrophysical and cosmological observations if their annihilation cross section is that of the weak interactions in the SM. The simplest WIMP models add a single particle to the SMthe WIMP itself-whereas more involved frameworks propose a whole new sector of particles, the lightest of which provides the DM. In the majority of the embedding models, the WIMP is a scalar, a fermion, or a vector particle, the annihilations into SM particles of which may occur through a Higgs-boson or Z-boson portal.

Assuming that the DM is a SM singlet, gauge-invariant renormalizable portals are possible for scalar or vector DM interacting via a Higgs portal. In the remaining cases, however, one must invoke an UV completion that allows for mixing with the SM particles or nonrenormalizable operators. We discuss many such possibilities in Sec. II. A. However, in order to assess in full generality the present viability of the Higgs-boson and Z-boson portals, here we treat all DM couplings as free parameters within a simple model-independent bottom-up approach. As in previous such analyses; see, e.g., Refs. [17,18], we disregard UVcompletion-dependent details concerning the origins of any higher-order operators and assume that any new states in the UV completions are so massive that they cannot impact the phenomenology. This approach allows us to discuss the phenomenology of all Higgs- and Z-boson-portal scenarios on the same footing and captures the essence of more involved UV constructions that recover the proposed setup in generic regions of their parameter spaces. 
Despite their simplicity, SM-portal models exhibit a rich phenomenology, with potential signals in direct and indirect detection (ID) experiments, as well as in collider searches for DM. The absence of such signals result in important constraints on their parameters, which were studied for scalar DM with a Higgs portal in Refs. [19-22]; for Higgs portals with DM of spin 0, $1 / 2$, and 1 in Refs. [23,24]; and for Higgs and $Z$ portals with DM of spin $0,1 / 2$, and 1 in Ref. [17]. The null results of the many searches have led to the suspicion that the plausibility of simple WIMP Higgs- and Z-portal models has been severely damaged; see, e.g., Refs. [18,25,26]. However, neither this nor the impact of planned DD experiments such as LZ [27] and XENONnT [28] has yet been quantified by a dedicated statistical analysis.

We address this point in this work by performing statistical analyses of the spin- $0,-1 / 2$, and -1 SM-portal models described in Sec. II. We use frequentist and Bayesian methodologies for the first time, jointly assessing the plausibility of these models. Our statistical framework is recapitulated in Appendix, and we add a novel determination of the number of constrained degrees of freedom at the end of Sec. V. Our work includes data from DD, ID, and collider experiments, and we also analyze the potential impacts of future DD experiments, using the pseudodata described in Sec. III. Our priors for the model parameters are presented in Sec. IV. Finally, we present our findings on the viability of SM-portal models in Sec. V and conclude in Sec. VI.

Unlike previous analyses, see, e.g., Refs. [17,18,25,26], which were ambivalent about the status of portal models, we find both Higgs- and Z-portal models that are entirely consistent with the available experimental constraints. These include Higgs-portal models with real scalar, real vector, Majorana, or Dirac fermion dark matter particles, as well as Z-portal models with Majorana or Dirac fermion dark matter particles. Many of the viable models feature relatively light dark matter particles that would contribute to invisible Higgs or $Z$ decays, and there are also interesting possibilities for heavier dark matter particles that weigh $\gtrsim 100 \mathrm{GeV}$. The planned direct dark matter search experiments would be sensitive to some of these dark matter models, but Higgs- and Z-portal models with Majorana or Dirac fermion dark matter particles could still escape detection. We ascribe the difference in emphasis between our paper and other analyses to the fact that we calculate statistical measures of the goodness of fit and relative plausibility of models; some previous analyses drew conclusions on the status of portal models without a clear statistical methodology. Our results open the way toward constructing viable DM models that are not significantly damaged by the present data. As with other simplified models, the models we discuss do not attempt to capture details and potential issues within any specific UVcomplete model. Although we discuss several viable UV completions in Sec. II. A in this simplified model approach, we do not include theoretical or experimental constraints upon the parameter spaces of any particular UV completions. The merit of this approach, which has proven extremely popular in DM (see, e.g., Refs. [17,19-24,2933]) and LHC studies (see, e.g., Refs. [34-41]), is that we may study the phenomenology of a comprehensive set of SM-portal models capturing common features.

\section{MODELS}

We consider simplified WIMP models in which spin-0, spin- $1 / 2$, or vector DM particles are stabilized by a $\mathbb{Z}_{2}$ symmetry, see, e.g., Ref. [13], and couple to the SM Higgs or $Z$ boson. This portal interaction with the SM has a number of phenomenological signatures. Through such portals, DM can scatter with quarks via $t$-channel exchanges producing signatures that are in principle observable at DD experiments. Portal couplings are also responsible for the annihilations of DM particles into SM particles, which control the relic abundance of DM via the freeze-out mechanism. The same DM annihilations could, furthermore, still be active in regions of space characterized by a high DM density, such as the Galactic center, dwarf spheroidal galaxies (dSphs), and possibly the Sun. Primary or secondary traces of annihilation could then be detected by ID experiments. In order to explore the vast phenomenology of these simplified models, we implemented them in FeynRules2.3.27 [42,43], linked with MICROMEGAs4.3.5 [44,45] via calcHEP [46].

\section{A. Higgs portal}

We consider models of spin- $0,-1 / 2$, and $-1 \mathrm{DM}$ particles coupling to the SM via a Higgs portal (see, e.g., Refs. [17,18] for similar Higgs-portal models), assuming that the DM is stabilized by a $\mathbb{Z}_{2}$ or $U(1)$ symmetry (see, e.g., Ref. [18]). Furthermore, in order to accommodate interactions between the Higgs and pairs of photons or gluons via loops, we include effective operators $h F_{\mu \nu} F^{\mu \nu}$ that result from the top quark and the $W$-boson loops in the SM:

(i) Dirac/Majorana fermion: DM particles interact with the SM via

$$
\mathcal{L} \supset c \bar{\chi}\left(g_{s}+i g_{p} \gamma^{5}\right) \chi h,
$$

where $g_{s}$ and $g_{p}$ are scalar and pseudoscalar couplings, respectively, and $c=1(1 / 2)$ for the Dirac (Majorana) case. We note that such interactions could originate from mixing after electroweak symmetry breaking (EWSB) between a SM singlet, $S$, and the Higgs in a gauge-invariant renormalizable model, as discussed in Ref. [47],

$\bar{\chi}\left(g_{s}+i g_{p} \gamma^{5}\right) \chi S \rightarrow \bar{\chi}\left(g_{s}+i g_{p} \gamma^{5}\right) \chi \sin \alpha h+\cdots$,

where $\alpha$ is a mixing angle between the Higgs and singlet. We assume that the singlet is so heavy that it cannot impact the phenomenology. Unitarity would require that [48] 


$$
\cos ^{2} \alpha m_{h}^{2}+\sin ^{2} \alpha m_{S}^{2} \lesssim(700 \mathrm{GeV})^{2},
$$

where $m_{h} \simeq 125 \mathrm{GeV}$ and $m_{S}$ is the mass of the singlet.

(ii) Scalar: DM particles interact with the SM via (see, e.g., Ref. [49])

$$
\mathcal{L} \supset c \lambda\left(v h|\chi|^{2}+\frac{1}{2} h^{2}|\chi|^{2}\right),
$$

where $c=1 / 2(1)$ for a real (complex) scalar and $v=246 \mathrm{GeV}$ is the vacuum expectation value of the SM Higgs. These interactions could result from a $|\chi|^{2}|H|^{2}$ operator after EWSB. Unitarity requires that $\lambda \leq 8 \pi[50]$.

(iii) Vector: $\mathrm{DM}$ interacts with the $\mathrm{SM}$ via

$$
\mathcal{L} \supset c g\left(v h \chi^{\mu} \chi_{\mu}^{\dagger}+\frac{1}{2} h^{2} \chi^{\mu} \chi_{\mu}^{\dagger}\right),
$$

where $c=1 / 2(1)$ for a real (complex) vector and $v=246 \mathrm{GeV}$ is the vacuum expectation value of the SM Higgs doublet. The vector DM may acquire a mass via the Stueckelberg mechanism (see, e.g., Ref. [51]). This interaction could arise by charging the SM Higgs under a new $U(1)$ gauge group, which would risk unacceptable mixing between the DM and SM $Z$ boson [52], or by an alternative mechanism (see, e.g., Refs. [53-55]), e.g., mixing between the SM Higgs and a SM singlet scalar that is charged under the $U(1)$.

All of these Higgs-portal models allow the possibility that there are no phenomenological signatures of the UV completions in current experiments, as we assume here.

\section{B. Z-boson portal}

We also consider all possible spin- $0,-1 / 2$, and $-1 \mathrm{DM}$ particles annihilating via a $Z$ portal:

(i) Dirac fermion: DM interacts with the $\mathrm{SM}$ via

$$
\mathcal{L} \supset \bar{\chi} \gamma^{\mu}\left(g_{v}+g_{a} \gamma^{5}\right) \chi Z_{\mu}
$$

where $g_{v}$ and $g_{a}$ are vector and axial couplings, respectively. This operator could originate after EWSB by coupling the fermion to the current $[56,57]$

$$
H^{\dagger} D_{\mu} H \rightarrow \frac{1}{2} i g v^{2} Z_{\mu}+\cdots
$$

via a nonrenormalizable operator, kinetic mixing between the SM $Z$ boson and a $Z^{\prime}$ boson [58], or if the DM has an $S U(2) \times U(1)$ charge [59]. The nonrenormalizable operator could, however, generate DM annihilation into $h h$ and $Z h$. Assuming that it originates from a nonrenormalizable operator, the interaction in Eq. (6) is unitary for couplings and masses of phenomenological interest [58]. (ii) Majorana fermion: DM interacts with the SM via

$$
\mathcal{L} \supset \frac{g_{a}}{2} \bar{\chi} \gamma^{\mu} \gamma^{5} \chi Z_{\mu} \text {. }
$$

The vector coupling is forbidden by charge conjugation for Majorana fermions as the operator is odd.

(iii) Scalar: DM interacts with the SM via

$$
\mathcal{L} \supset i g \chi^{\dagger} \stackrel{\leftrightarrow}{\partial}^{\mu} \chi Z_{\mu}+g^{2}|\chi|^{2} Z^{\mu} Z_{\mu},
$$

where $a \stackrel{\leftrightarrow}{\partial}^{\mu} b:=a\left(\partial^{\mu} b\right)-b\left(\partial^{\mu} a\right)$ and $g$ is an effective gauge coupling. These operators may originate from kinetic mixing between the SM $Z$ boson and a $Z^{\prime}$ boson [58] or via higher-order terms of the form

$$
\mathcal{L} \supset g^{\prime}\left(\chi^{\dagger} \stackrel{\leftrightarrow}{\partial}^{\mu} \chi\right) \frac{H^{\dagger} \stackrel{\leftrightarrow}{D}_{\mu} H}{\mu^{2}} .
$$

(iv) Vector: DM interacts with the $\mathrm{SM}$ via

$$
\mathcal{L} \supset i g\left(Z^{\mu} \chi^{\nu \dagger} \partial_{[\mu} \chi_{\nu]}+\chi_{\mu}^{\dagger} \chi_{\nu} \partial^{\mu} Z^{\nu}\right)+\text { H.c. }
$$

where $g$ is an effective gauge coupling (see, e.g., Ref. [18]). The higher-order operators that can induce these interactions are rather involved; we refer the reader to Refs. [60-63] for possible realizations. As in the Higgs-portal case, we assume here that the vector DM acquires a mass via the Stueckelberg mechanism.

We note that the couplings of the $Z$ boson to particles in specific representations of the SM gauge group must take discrete values in terms of the $\mathrm{SU}(2)$ and $\mathrm{U}(1)$ gauge couplings. However, more general values are allowed in the presence of mixing between the DM and SM particles. In the interest of generality, we allow for this possibility without entering into specific mixing scenarios. We note also that in the spin- $1 / 2$ and -1 cases there are unitarity issues (anomaly cancellation and rapid increase in scattering amplitudes at high energies, respectively) that need to be addressed in the UV completion (see e.g., Ref. [56]), the possible experimental signatures of which we do not discuss here.

\section{LIKELIHOOD}

The likelihood function is a critical ingredient for our frequentist and Bayesian statistical methodologies, which are described in Appendix. Our likelihood, summarized in Table VII, includes collider, ID, and DD searches for DM, as well as the Planck determination of the DM relic abundance. Our implementations of the likelihood contributions required are detailed in the forthcoming sections. As explained in Secs. IIIC and III D, we approximate experimental limits on DD and ID cross sections by step functions, similar to the treatment in, e.g., Ref. [18]. We, 
however, incorporate important nuclear and astrophysical uncertainties.

\section{A. Relic density}

We assume that the DM candidate accounts for all of the observed DM; i.e., it is not an underabundant species of DM. We calculate the relic abundance of DM with MICROMEGAS4.3.5. We describe the Planck determination of the relic density [1] by a Gaussian and include in quadrature a supplementary $10 \%$ theoretical uncertainty in the calculation of the relic abundance. Recent analyses suggest that the standard treatment in MICROMEGAs4.3.5 may be insufficient, as it neglects the $s$-dependence of the mediator width in a Breit-Wigner and the effect of kinetic decoupling $[64,65]$, but we leave any improvement to future work.

\section{B. Collider constraints}

\section{Invisible decay widths}

The invisible width of the $Z$ boson was measured by large electron-positron collider (LEP) [66]. We calculate the SM contribution, due to $\Gamma(Z \rightarrow \nu \nu)$, with a parametric two-loop formula from Ref. [67], which includes the dependence on $M_{Z}$, which we treat as a nuisance parameter. We calculate $\Gamma(Z \rightarrow$ DM $)$ with MICROMEGAs4.3.5 and implement the measurement as a Gaussian upon $\Gamma(Z \rightarrow \nu \nu)+\Gamma(Z \rightarrow$ $\mathrm{DM})$. The branching fraction of a SM-like Higgs to invisible particles was constrained by CMS [68] to be $\operatorname{BR}(h \rightarrow$ invisible $) \lesssim 0.24$. Since the likelihood function has been published, we apply it directly in our analysis. We neglect any correlations between measurements of the $\mathrm{SM}$ masses and widths, and we neglect $\mathrm{BR}(h \rightarrow \nu \nu)$, which is absent in the SM.

\section{Monojet and monophoton searches}

Monojet and monophoton searches look for missing transverse momentum resulting from DM particles produced in pairs that escape the detector. The DM particles are not produced back to back in the laboratory frame when they recoil from the emission of a jet or photon. We include $\sqrt{s}=$ $8 \mathrm{TeV}$ and $13 \mathrm{TeV}$ monojet and monophoton searches for DM at the LHC via CHECKMATE2[69-74]. The searches that we included are listed in Table I, and the limits we use are shown in Fig. 1. In the models under study, production cross sections at $\sqrt{s}=8 \mathrm{TeV}$ and $13 \mathrm{TeV}$ are comparable, and the greater integrated luminosity in the former make them more powerful. ${ }^{1}$ Since we add to the SM only a DM particle, there are no cascade decays of dark sector particles resulting in, e.g., jets or leptons.

\footnotetext{
${ }^{1}$ We did not include a recent ATLAS monojet search at $\sqrt{s}=$ $13 \mathrm{TeV}$ with $36 \mathrm{fb}^{-1}[75]$ that is not yet implemented in CHECKMATE2. We do not anticipate that this would change significantly the results we find using the monojet searches that are implemented in CHECKMATE2.
}

TABLE I. Monojet and monophoton LHC searches for DM included in our analysis via CHECKMATE2.

\begin{tabular}{lrrr}
\hline \hline Analysis & $\sqrt{s}(\mathrm{TeV}) \int \mathcal{L}\left(\mathrm{fb}^{-1}\right)$ & CHECKMATE2 \\
\hline ATLAS monojet [6] & 8 & 20.3 & ATLAS_1407_0608 \\
ATLAS monojet [7] & 8 & 20.3 & ATLAS_1502_01518 \\
ATLAS monojet [8] & 13 & 3.2 & ATLAS_1604_07773 \\
CMS monojet [5] & 8 & 19.7 & CMS_1408_3583 \\
ATLAS monophoton [10] & 8 & 20.3 & ATLAS_1411_1559 \\
ATLAS monophoton [11] & 13 & 3.2 & ATLAS_1604_01306 \\
ATLAS monophoton [9] & 13 & 36.1 & ATLAS_1704_03848 \\
\hline \hline
\end{tabular}

We generated events from our models with MADGRAPH5_AMC@NLO[77] and processed the resulting LHE event files with CHECKMATE2. We derived our own 95\% exclusion contours on planes of mass versus coupling for each SM-portal model for the ranges of mass and coupling in Table VIII. We approximated the likelihood function by a step function by assigning to each parameter point a likelihood of zero if it was excluded at $95 \%$ and 1 otherwise.

\section{Direct detection}

We include the world-leading SI DD constraints from PandaX [4] and world-leading SD DD constraints for neutrons from PICO [2] and for protons from PandaX [78]. We have calculated scattering cross sections with MICROMEGAS4.3.5 while noting, however, that there are appreciable uncertainties that we now discuss and have considered in our analysis.

The WIMP interactions with partons can be described by an effective field theory. For nonrelativistic velocities, scalar, vector, pseudovector, and tensor operators dominate, as other operators are velocity suppressed. The scalar, vector, pseudovector, and tensor operators contribute to SI even, SI odd, SD even, and SD odd interactions, respectively [79]. The WIMP interactions with nucleons, which are relevant for $\mathrm{DD}$, are governed by the partonic interactions and nuclear form factors. The vector, pseudovector, and tensor form factors are well known from lattice calculations and because the vector form factor depends only upon the valence quarks. We set them to their MICROMEGAS4.3.5 defaults.

However, there are uncertainties in the scalar form factors, which depend upon

$$
\begin{gathered}
\sigma_{s} \equiv m_{s}\langle N|s \bar{s}| N\rangle, \\
\sigma_{\pi N} \equiv \frac{1}{2}\left(m_{u}+m_{d}\right)\langle N|u \bar{u}+d \bar{d}| N\rangle,
\end{gathered}
$$

as well as the ratios of light-quark masses. There is tension between determinations of $\sigma_{\pi N}$ : lattice determinations favor about $40 \mathrm{MeV}$ [80], whereas phenomenological determinations favor about $60 \mathrm{MeV}$ [81-84]. We modify the 

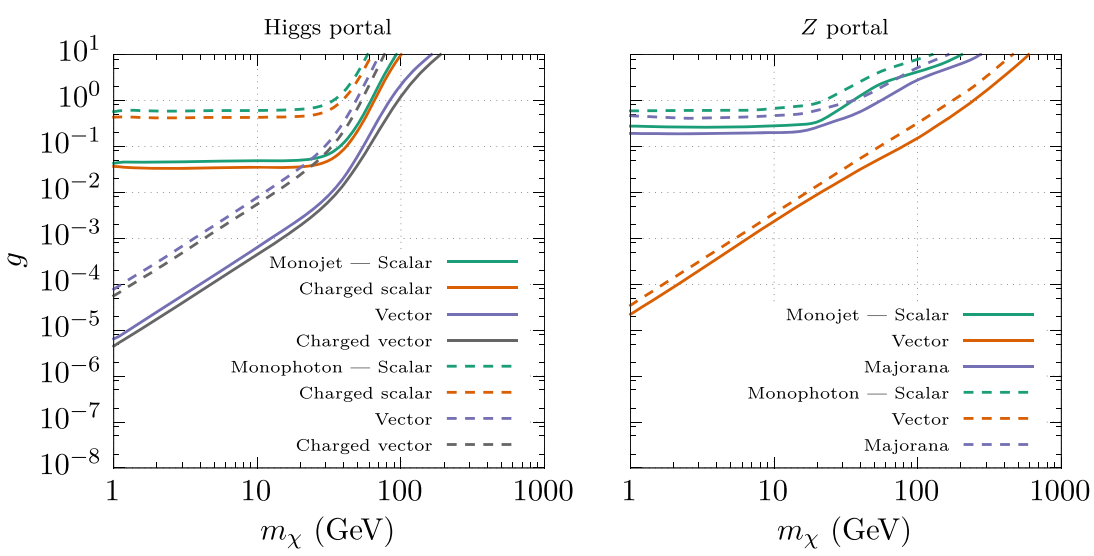

FIG. 1. Exclusion limits from LHC monojet (solid lines) and monophoton (dashed lines) searches for DM in the Higgs- (left) and $Z$-portal (right) models, which have a single coupling $g$ to the SM.

MICROMEGAS4.3.5 default of $34 \mathrm{MeV}$, picking a prior that is flat between a precise lattice determination of $37.2 \mathrm{MeV}$ [85] and a recent phenomenological determination of $58 \mathrm{MeV}$ [84], with Gaussian tails equal to the experimental uncertainties. To check the sensitivity of our results to this treatment, we perform extra calculations in which we discard lattice results and use only the phenomenological determination. We pick a Gaussian for $\sigma_{s}$ from a lattice determination $[85]^{2}$ and flat priors between intervals reported by the PDG [66] for ratios of light-quark masses. Even though a recent analysis [86] indicates that uncertainties in the Higgs-nucleon coupling are likely to be overestimated, for our analysis, we choose a conservative approach in our treatment of the DD data.

In addition to nuclear uncertainties, there are astrophysical uncertainties that affect DD, such as the velocity profile and local density of DM. We include a log-normal uncertainty upon a canonical choice of local density, $\rho=0.3 \mathrm{GeV} / \mathrm{cm}^{3}$. Since we assume that the DM accounts for the entire relic abundance, we do not rescale DD cross sections by, e.g., $\Omega h^{2} / 0.1$. For all but the real scalar Higgs portal, we neglect velocity profile uncertainties. For that model, though, we vary the shape parameters of a truncated Maxwell-Boltzmann velocity distribution describing the velocity profile.

In our implementation of the DD likelihood, we picked a step function at the $90 \%$ limits for the scattering cross section from PandaX and PICO for a particular DM mass and marginalized uncertainties in the local DM density, nuclear form factors, and ratios of light-quark masses. As a cross-check, in specific cases, we used the likelihood functions implemented at the event level in DDCALC1.1.0

\footnotetext{
${ }^{2}$ An alternative approach estimates $\sigma_{s}$ using $\sigma_{\pi N}$ and phenomenological estimates of the SU(3)-breaking contribution to baryon masses, $\sigma_{0}$. However, this is subject to considerable uncertainties in $\sigma_{0}$ [82] as well as $\sigma_{\pi N}$.
}

[87] and marginalized the local DM density and velocity distribution.

To the best of our knowledge, since we investigated the DM velocity profile, this constitutes the most comprehensive treatment of DD uncertainties to date. In, for example, a recent GAMBIT analysis of DD in the real scalar $h$ portal [21], only the light-quark masses, the local density, and form-factor uncertainties were treated with nuisance parameters. Note, moreover, that our prior of $\sigma_{\pi N} \approx 27-58 \mathrm{MeV}$ differs from that in GAMBIT of $58 \pm 9 \mathrm{MeV}$, reflecting the tension between the lattice and phenomenological determinations.

\section{Indirect detection}

We consider the Fermi-LAT limit from several dSphs [88] on the zero-velocity limit of the DM annihilation cross section. As in the case of DD cross sections, we do not rescale ID cross sections by, e.g., $\Omega h^{2} / 0.1$ as we assume that the DM accounts for the entire relic abundance. We approximate the likelihood function by step functions at the $95 \%$ exclusion contours on the $(m,\langle\sigma v\rangle)$ planes for $u \bar{u}, b \bar{b}$, $W W, e \bar{e}, \mu \bar{\mu}$, and $\tau \bar{\tau}$ channels. The astrophysical uncertainties for ID are characterized by uncertainties in $J$ factors for each dSph. Since we consider Fermi-LAT's combined limit from several dSphs, we make an approximation: we model uncertainties in the limit by a universal $J$-factor uncertainty that scales the whole signal, picking an uncertainty in $\log _{10} J$ of 0.25 motivated by typical $J$-factor uncertainties for individual galaxies. Of the $15 \mathrm{dSphs}$ included in the Fermi-LAT limit, the greatest $\log _{10} J$ uncertainty was 0.31 , and the smallest was 0.16 . Since a universal $J$-factor implies that the uncertainties are $100 \%$ correlated, we anticipate that our treatment is conservative (i.e., overestimates the uncertainties). We could, in principle, perform a detailed treatment of the Fermi-LAT data with 15 individual $J$-factors. For the portal models under consideration, however, the ID cross sections are typically 

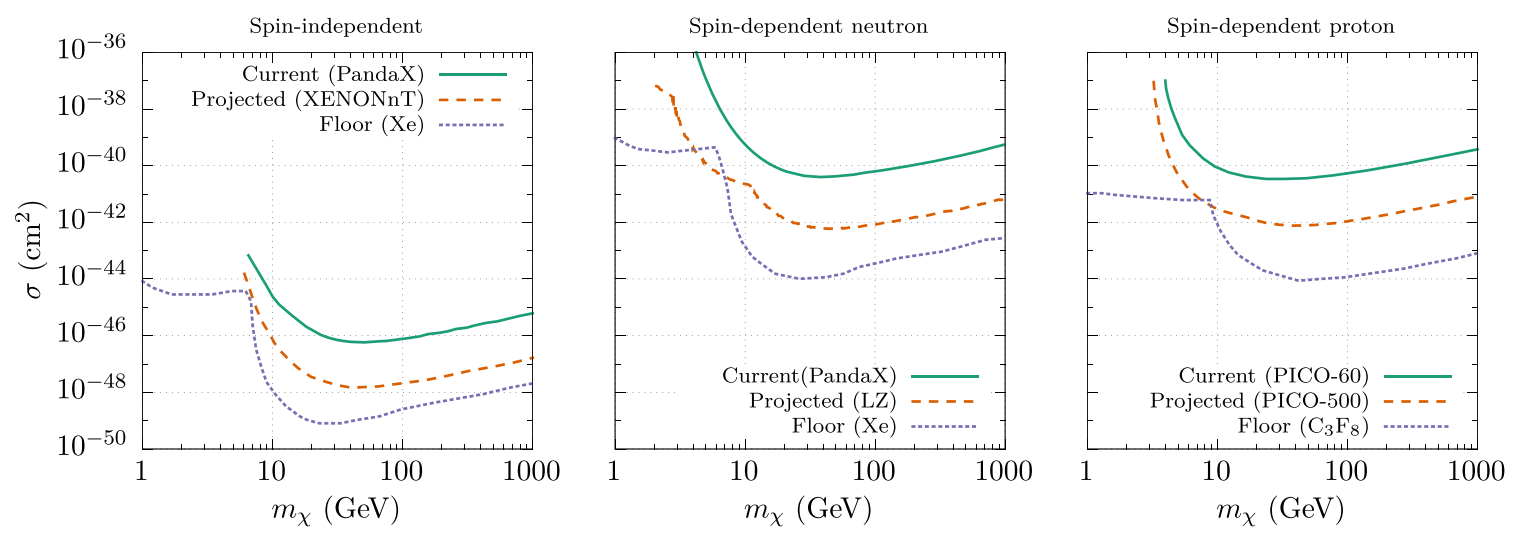

FIG. 2. Current (green solid lines), projected (orange dashed lines), and neutrino floor (blue dotted lines) $90 \%$ limits for DD searches for DM in spin-independent (left), spin-dependent neutron (center), and spin-dependent proton interactions. See Table VII for further details.

smaller than the limits from Fermi-LAT, and we argue that our simplified treatment is adequate.

We do not include constraints from solar neutrinos from IceCube [89]. IceCube may compete with PICO limits on the spin-dependent scattering cross section, though this interpretation requires assumptions about DM spinindependent interactions in the Sun, the square of the local density, whether DM reaches equilibrium in the Sun, and the rates at which DM annihilates to specific final states. We anticipate that a careful treatment of uncertainties, as discussed in Ref. [90], would render the IceCube limit weaker than or similar to that from PICO in our models.

\section{E. Future data}

In assessing the potential impacts of future experiments, we utilize projected 90\% exclusions from LZ [91], XENONnT [28], and PICO500 [92] and an estimate of the neutrino floor-the level of scattering cross section at which a WIMP signal becomes hard to distinguish from the neutrino background [93]. The neutrino floors assume Xe targets, except for the spin-dependent (SD) interaction with protons, which assumes a $\mathrm{C}_{3} F_{8}$ target, as in the PICO experiment. The neutrino floor can be very slowly lowered with greater exposure (or overcome with, e.g., directional detection, modulation, or complementarity between different target nuclei); see Ref. [93] for details of the assumed exposures. The current limits, projected limits, and neutrino floors are shown in Fig. 2.

The projected limits are expected limits assuming the background only hypothesis, i.e., no DM. The observed limit would, however, be subject to statistical fluctuations. We do not investigate the impact of such fluctuations and assume that evidences calculated with projected limits are similar to ones for which the fluctuations are averaged. We anticipate that this is reasonable to within the desired uncertainty of about an order of magnitude in the evidence.

\section{PRIORS}

The priors for the model parameters used in our Bayesian analysis are shown in Table VIII. In addition to the DM mass and couplings, there are numerous nuisance parameters describing uncertainty about, e.g., the local density of DM, as discussed in Sec. III. Our priors for the DM mass and couplings are quite relaxed; we permit masses between 1 and $10^{4} \mathrm{GeV}$ and couplings between $10^{-6}$ and $4 \pi$. Since we are a priori ignorant of their scale, we pick logarithmic priors for the mass and couplings, e.g., $p(\ln m \mid M)=$ const. For models with two couplings, we pick factorizable priors, reflecting the assumption that the couplings are determined by different mechanisms and thus independent, e.g., $p\left(g_{s}, g_{p} \mid M\right)=p\left(g_{s} \mid M\right) \times p\left(g_{p} \mid M\right)$. We investigate the sensitivity to priors in Sec. V C.

\section{RESULTS}

We interfaced our private code to (Py-)MultiNeST3.10 [94-97] and analyzed our data with SUPERPLOT [98]. We used 5000 live points and a stopping tolerance of $10^{-4}$ and selected importance sampling determinations of evidences and posteriors.

We show Bayes factors and minimum $\chi^{2}$-values for our SM-portal models in Table II, considering all present data. The most successful is the Majorana Z-portal model, but several of the other models are also highly consistent with the available data, as indicated by their Bayes factors, minimum $\chi^{2}$, and $p$-values. These are the $h$-portal models with scalar, vector, or fermionic DM and the $Z$-portal models with fermionic DM. On the other hand, two of the models have calculated Bayes factors that should be interpreted as "decisive" evidence against them. These are the scalar and vector $Z$-portal models. Given the available data, the scalar and vector $Z$-portal models are about $10^{-14}$ and $10^{-10}$ less plausible than other SM-portal models. The models are essentially falsified by DD 
TABLE II. Bayes factors, $\chi^{2}$-values, and $p$-values for DM models in light of DD experiments in addition to measurements of the relic abundance and collider and indirect detection data. The color scheme for the Bayes factors reflects interpretation on the Jeffreys' scale for negative evidence [76]: green indicates barely worth mentioning; orange indicates substantial evidence; and red indicates strong, very strong, or decisive evidence. The Bayes factors are relative to the best model (the Majorana Z-portal). Similar colors are used for the interpretations of the $p$-values calculated within the frequentist approach.

\begin{tabular}{lccc}
\hline \hline Model & Bayes factor & Min. $\chi^{2}$ & $p$-value \\
\hline Real scalar $h$ portal & 0.55 & 2.6 & 0.27 \\
Complex scalar $h$ portal & 0.28 & 2.6 & 0.27 \\
Real vector $h$ portal & 0.23 & 2.6 & 0.27 \\
Complex vector $h$ portal & 0.059 & 2.6 & 0.27 \\
Majorana $h$ portal & 0.59 & 2.6 & 0.27 \\
Dirac $h$ portal & 0.71 & 2.6 & 0.27 \\
Scalar $Z$ portal & $3 \times 10^{-14}$ & 55 & $1.4 \times 10^{-12}$ \\
Vector $Z$ portal & $6.8 \times 10^{-10}$ & 35 & $2.2 \times 10^{-8}$ \\
Majorana $Z$ portal & 1 & 2.6 & 0.27 \\
Dirac $Z$ portal & 0.24 & 2.6 & 0.27 \\
\hline \hline
\end{tabular}

searches, as they predict relatively large SI scattering cross sections on nucleons.

As for the minimum $\chi^{2}$-values, we find that in most of the models there is sufficient freedom to minimize this quantity, barring for a small contribution from the invisible width of the $Z$ boson (the observed invisible width is slightly less than the SM prediction). This results in a common baseline for the minimum $\chi^{2}$-value of about 2.6. $\mathrm{DD}$, however, impairs the scalar and vector $Z$-portal models, inducing total $\chi^{2}$-values of about 54 and 35 , respectively. With 2 degrees of freedom, these correspond to $p$-values of about $10^{-12}$ and $10^{-8}$, respectively.

We display in Figs. 3-6 the confidence regions found in the frequentist analysis of the models that were not decisively disfavored. The credible regions in our Bayesian analysis (not shown) are similar. In each case, we indicate in (only ten) shades of gray the relic density calculated using MICROMEGAs4.3.5, and we outline with solid (dashed) red contours the regions preferred at the 1and 2- $\sigma$ levels. In general, there are two regions of model parameter space of interest: one in which on-shell portal particles can decay directly into pairs of on-shell DM particles and another in which the DM particles can be produced only via off-shell portal particles. As seen in Fig. 3, both of these are open possibilities in the Higgsportal model with real scalar DM. Panel (a) shows results with only the relic density and collider constraints, panel (b) includes also the indirect DM detection constraint, panel (c) further includes the current direct DM detection constraints, and panel (d) shows the impact of a possible null result from the LZ experiment (the impact of a null result from the XENON1T experiment would be very similar).

The upper panels of Fig. 4 compare the confidence regions for Higgs-portal models with (a) real and (b) complex scalar DM, incorporating all the present constraints.
We see that the results are quite similar. We see in the lower panels of Fig. 4 that the same is true for the confidence regions for Higgs-portal models with (c) real and (d) complex vector DM. In these models, there are also two confidence regions, corresponding to on- and off-shell Higgs couplings to the DM particles.

Figure 5 displays the confidence regions for Higgs-portal models with fermionic DM in light of all present data. The upper panels are for Dirac fermions, and the lower panels are for Majorana fermions. In each case, there are two couplings to the Higgs: scalar $g_{s}$ (left panels) and pseudoscalar $g_{a}$ (right panels). There are extended regions of the scalar couplings with $\log _{10} g_{s}<-1.5$ that are favored at the 1- $\sigma$ level, whereas there are only narrow confidence bands with $\log _{10} g_{p}>-1.5$ in the pseudoscalar cases. The DM may annihilate through a combination of scalar and pseudoscalar couplings. The scalar coupling is forced to be small by DD constraints, whereas the pseudoscalar coupling is not affected by DD constraints as its contributions to the scattering cross section are momentum suppressed. Since the scalar coupling must be small, the pseudoscalar coupling must set the correct relic density and so is much more restricted. In these models, the only confidence regions correspond to off-shell Higgs couplings to $\mathrm{DM}$ particles weighing $\gtrsim 100 \mathrm{GeV}$. The Bayes factors, minimum $\chi^{2}$, and $p$-values for these Higgs-portal models are all quite comparable, as seen in Table II, with insignificant evidence against any of them.

On the other hand, the same table shows that there is decisive evidence against the scalar and vector $Z$-portal DM models, and we do not display the mass/coupling planes for these models. We do, however, display the corresponding planes for Z-portal fermionic DM models in Fig. 6. The upper panels are for Dirac fermion DM, and the lower panel is for Majorana fermion DM. In the former case, we consider both axial (upper left) and axial (upper right) 


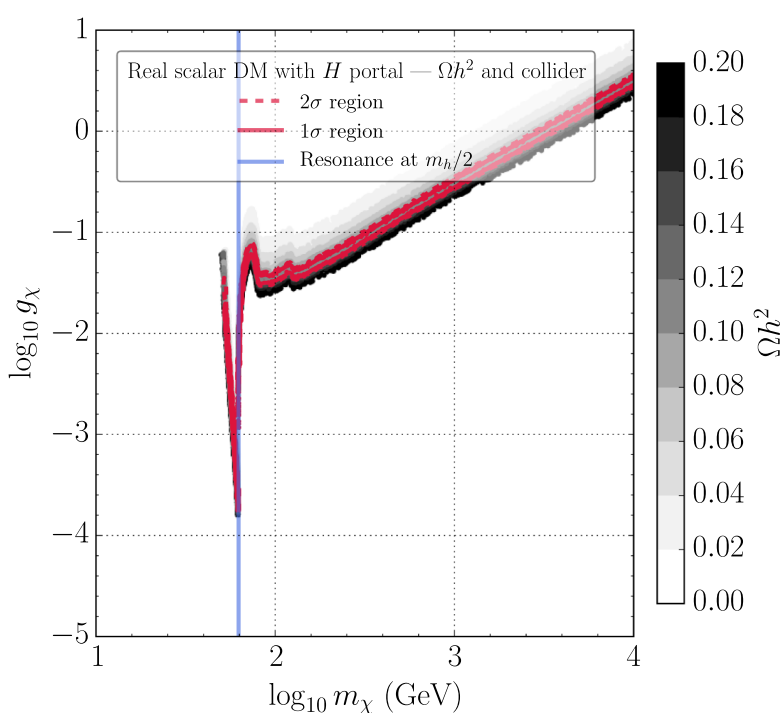

(a) Relic density and collider constraints.

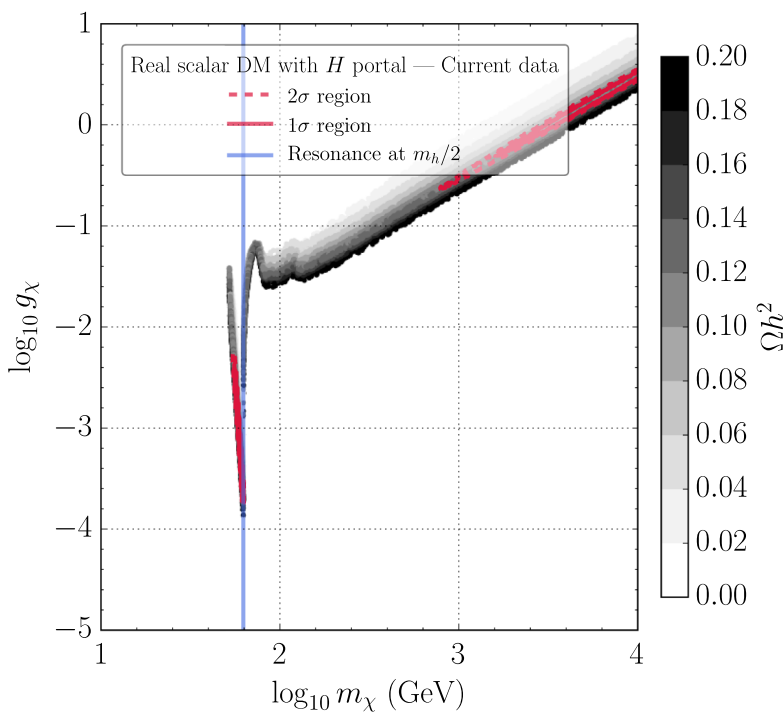

(c) Direct and indirect detection, relic density and collider constraints.

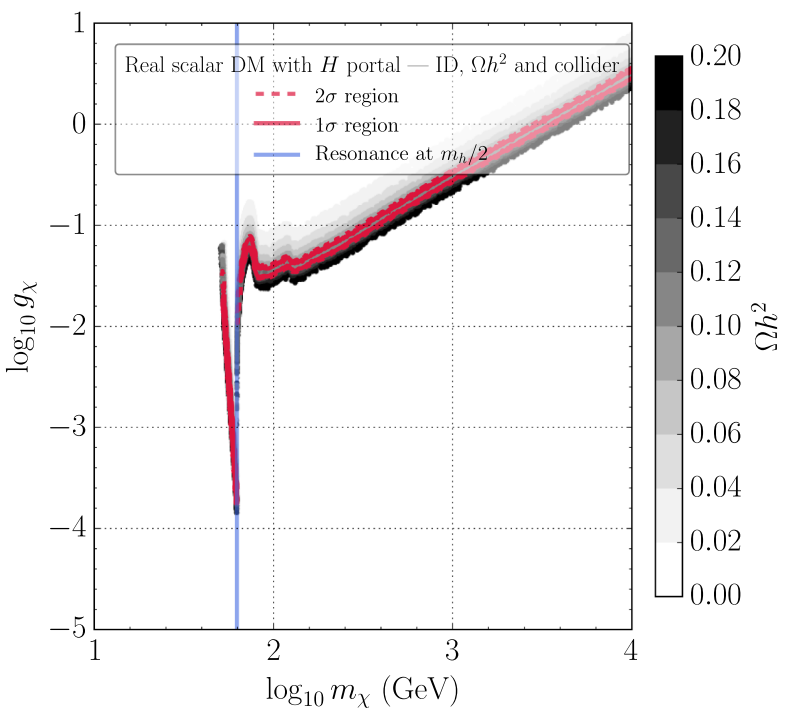

(b) Indirect detection, relic density and collider constraints.

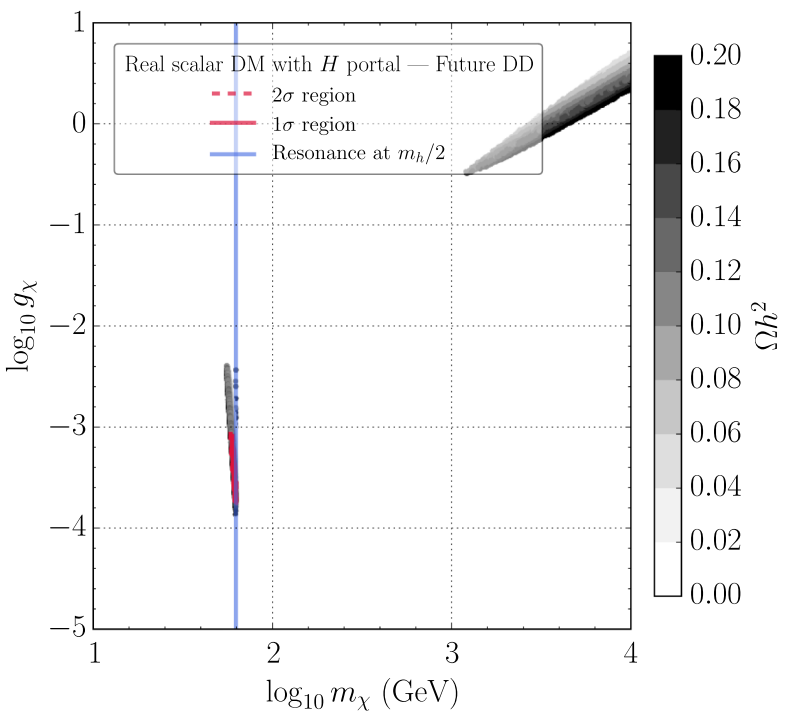

(d) Possible LZ constraints on direct detection, indirect detection, relic density and collider constraints.

FIG. 3. Confidence regions of DM mass and coupling for real scalar Higgs-portal DM in light of data from various experiments.

DM- $Z$ couplings, whereas in the Majorana case, only an axial DM- $Z$ coupling is allowed. In all three cases, only the off-shell $Z$ option with a DM particle mass $\gtrsim 200 \mathrm{GeV}$ is credible. As seen in Table II, the Majorana $Z$-portal DM model currently has the largest Bayes factor and also shares with the Dirac $Z$-portal case the lowest $\chi^{2}$ minimum and the highest $p$-value.

In Table III, we show partial Bayes factors (PBFs) for DD data for SM-portal models versus a hypothetical DM model with no DD signatures. This illustrates the damage to DM models from DD data. We consider present DD limits from PandaX and PICO, projections of possible future DD limits from LZ and PICO, and the neutrino floor for spin-independent limits. The projected DD limits would damage the plausibility of Higgs-portal models. Except for the fermion-portal models, the damage to their plausibility shifted from "barely worth mentioning" to "substantial." The statuses of the Z-portal models were unchanged, although the scalar and vector models were further damaged. Taking the spin-independent (SI) limits on DD cross sections to the neutrino floor would provide decisive evidence against all scalar and vector SM portals 


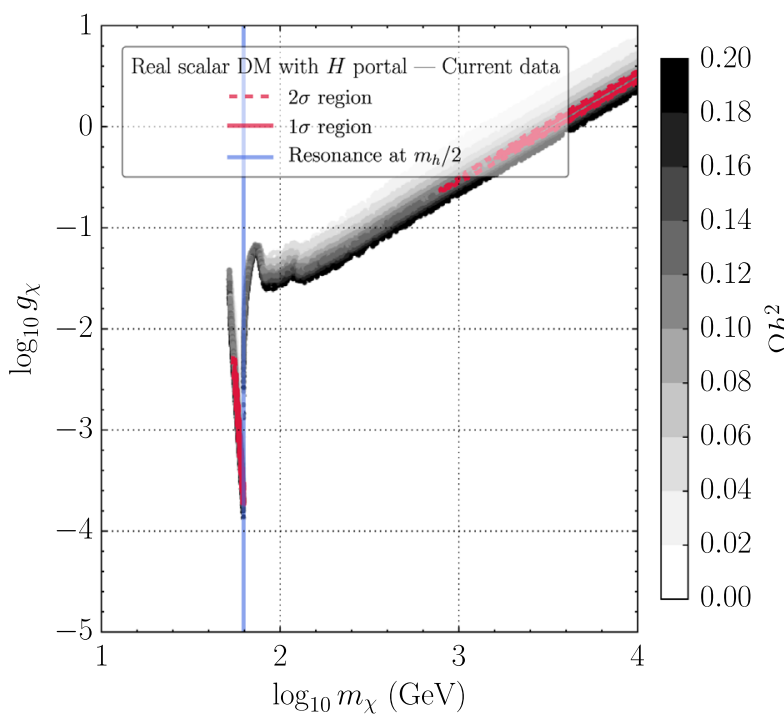

(a) Real scalar, all present constraints

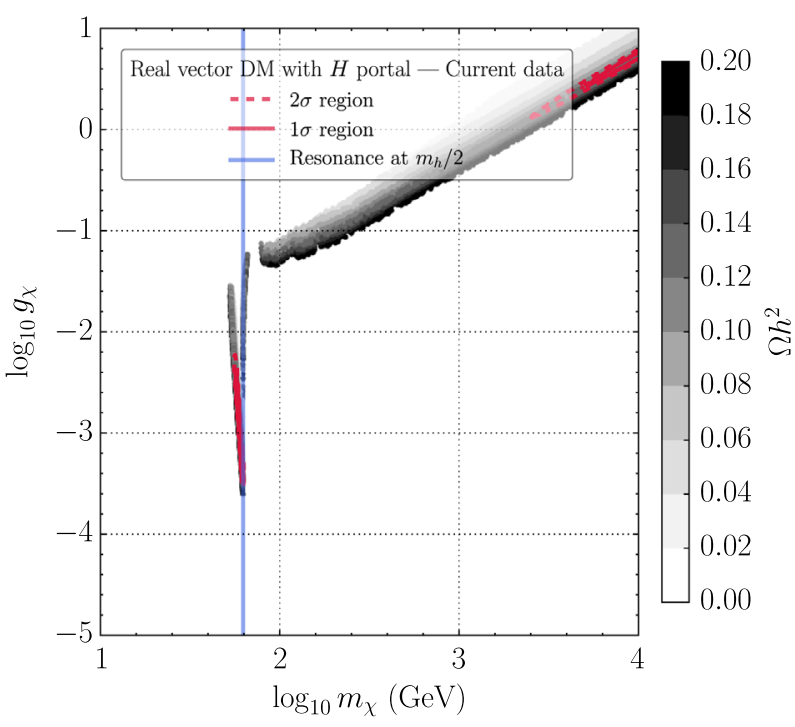

(c) Real vector, all present constraints

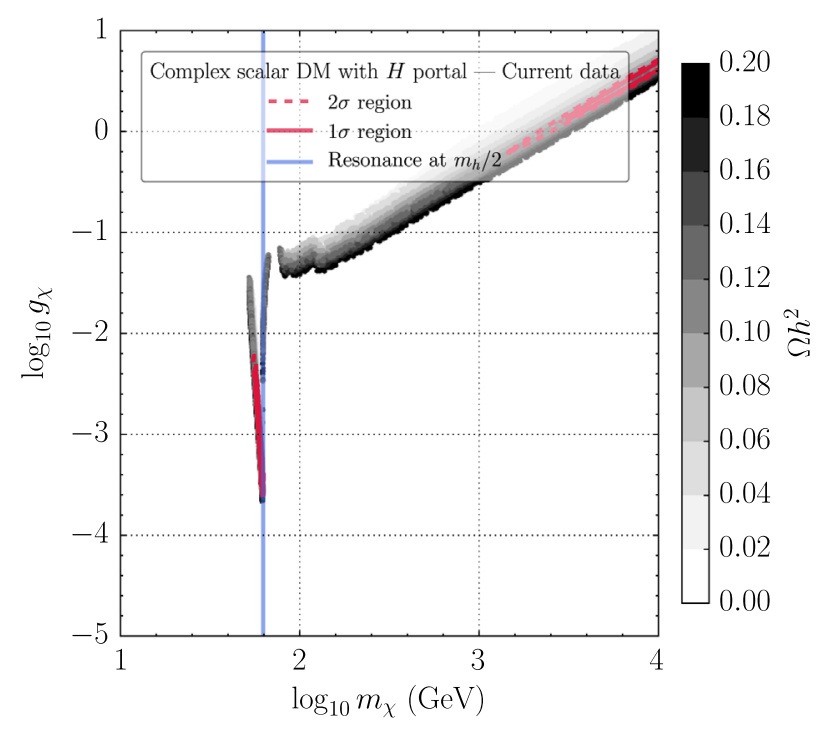

(b) Complex scalar, all present constraints

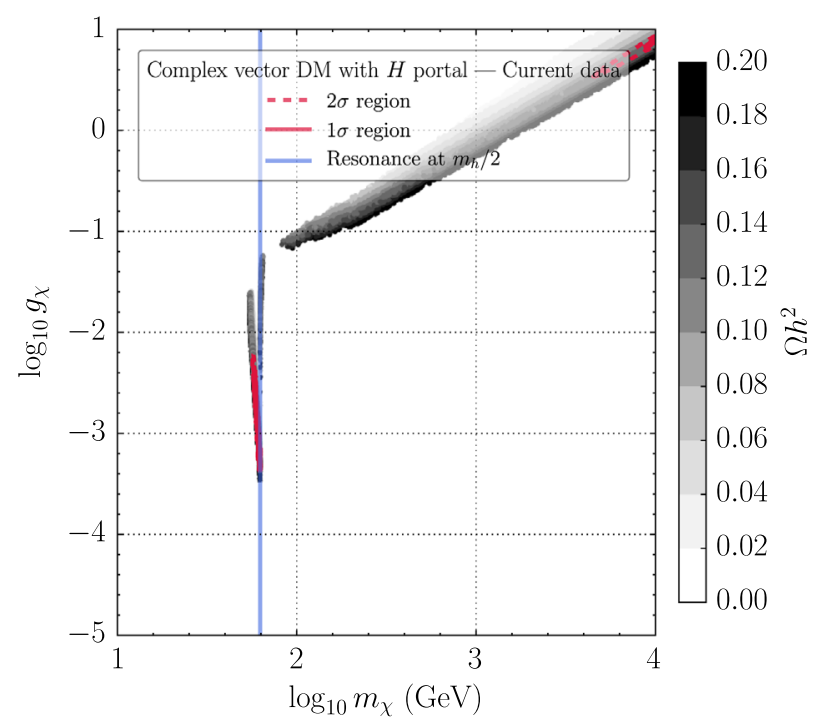

(d) Complex vector, all present constraints

FIG. 4. Confidence regions of DM mass and coupling for Higgs-portal scalar and vector DM models in light of all present data.

if no signal were observed, but fermion portals would not be harmed.

We compare the findings from the Bayes factors with the corresponding frequentist analysis in Table IV. The $\chi^{2}$-values for the scalar and vector $Z$ portals increase substantially when possible future data are considered. The statuses of the remaining models barely change, however, though the vector Higgs portal might be ruled out by SI limits at the neutrino floor. Even for our simple SM-portal models, we can fine tune the mass and coupling to evade possible powerful constraints upon the DD cross section from future experiments. Fine-tuned masses and couplings can enhance DM annihilation by an $s$-channel resonance, i.e.,

$$
\sqrt{s} \approx 2 m_{\chi} \simeq M_{Z} \quad \text { or } \quad m_{h}
$$

Elastic scattering on nucleons, though, proceeds via $t$-channel exchange and is not enhanced.

\section{A. DD uncertainties}

We find that for the impact of uncertainties in DD evidences are small. The uncertainties smear a limit in the scattering cross section, as in Fig. 7. While this smearing affects the posterior density, the evidence, which is an average likelihood, is relatively stable with respect to hadronic, velocity profile, and density uncertainties. 


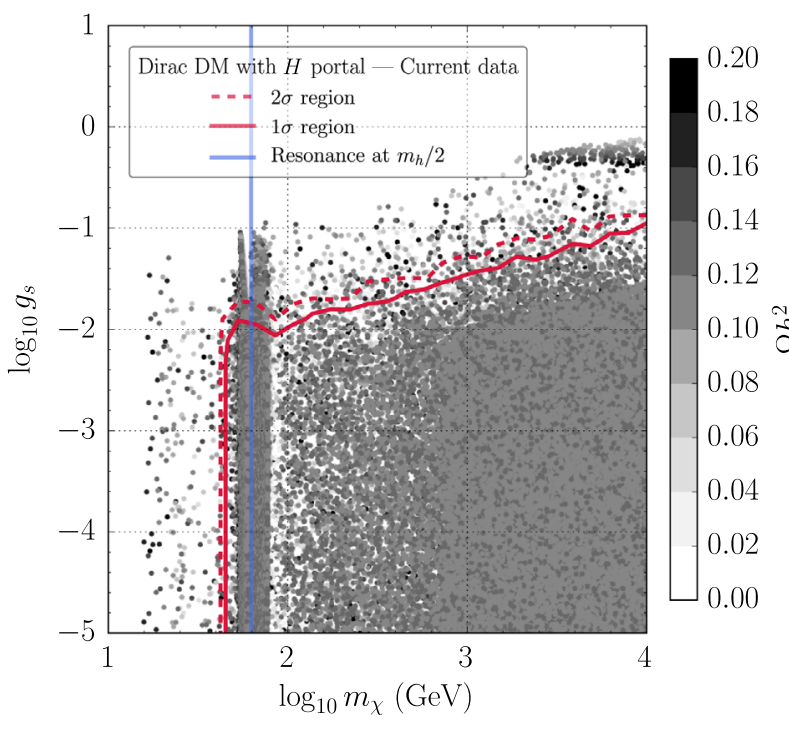

(a) Dirac DM, scalar coupling

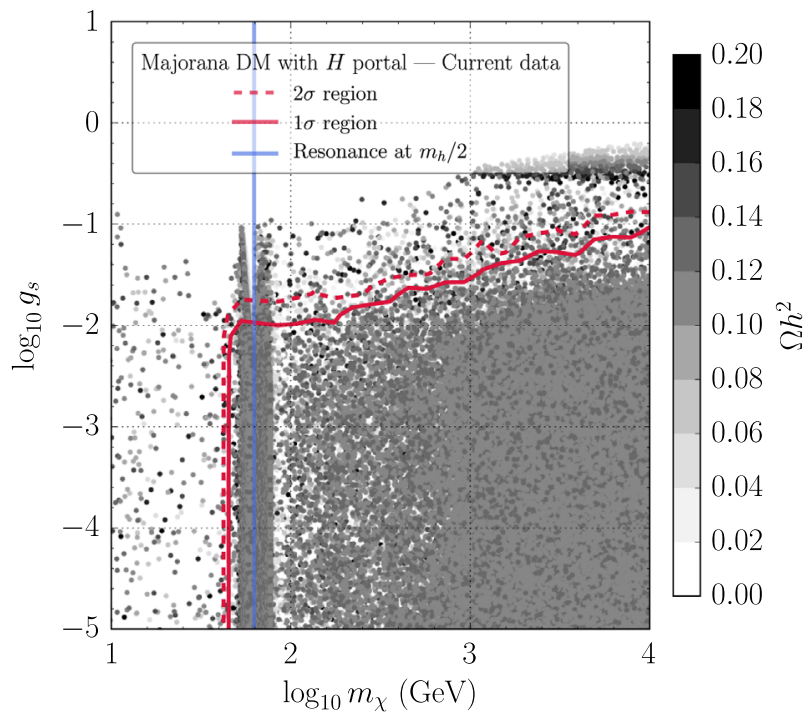

(c) Majorana DM, scalar coupling

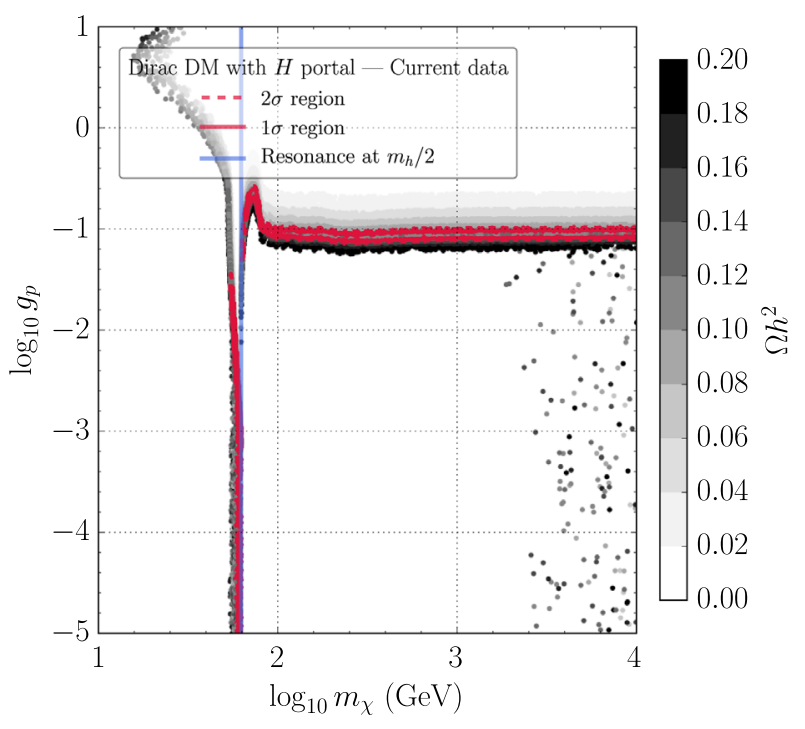

(b) Dirac DM, pseudoscalar coupling

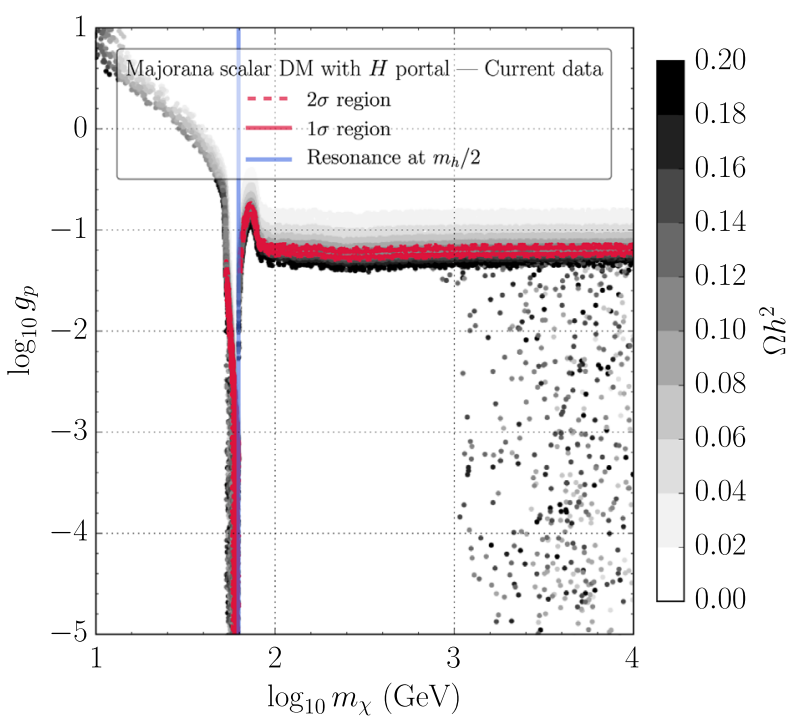

(d) Majorana DM, pseudoscalar coupling

FIG. 5. Confidence regions of DM mass and coupling for Higgs-portal fermionic DM models in light of all current data.

In Table V, we show evidences for the real scalar Higgsportal model with DD, ID, relic abundance, and collider data, computed with our native implementation of a likelihood for DD and that from DDCALC1.1.0, with different uncertainties included. DDCALC1.1.0 includes XENON1T data, which are fractionally weaker than PandaX for larger DM masses.

Uncertainties that change Bayes factors by less than about a factor of 5 are typically considered irrelevant, since they are overwhelmed by changes in Bayes factors induced by changes of priors. The changes from treatments of DD uncertainties in Table $\mathrm{V}$ are of order unity, and hence largely irrelevant. The biggest impact is that from including the uncertainty in the local density in our calculation of the likelihood, which smears a step function to a Gaussian error function as shown in Fig. 7, bringing it closer toward the DDCALC1.1.0 likelihood. The difference between our implementation of DD constraints and that in DDCALC1.1.0 results in a factor of order 1 in evidence. Including velocity profile uncertainties, which we omitted elsewhere, changes the evidence by a factor of about 3, which may be considered irrelevant.

\section{B. Treatment of resonances}

To ensure that narrow resonances are adequately sampled, for the real scalar Higgs-portal model, we investigated our sampling settings. We varied the number of live 


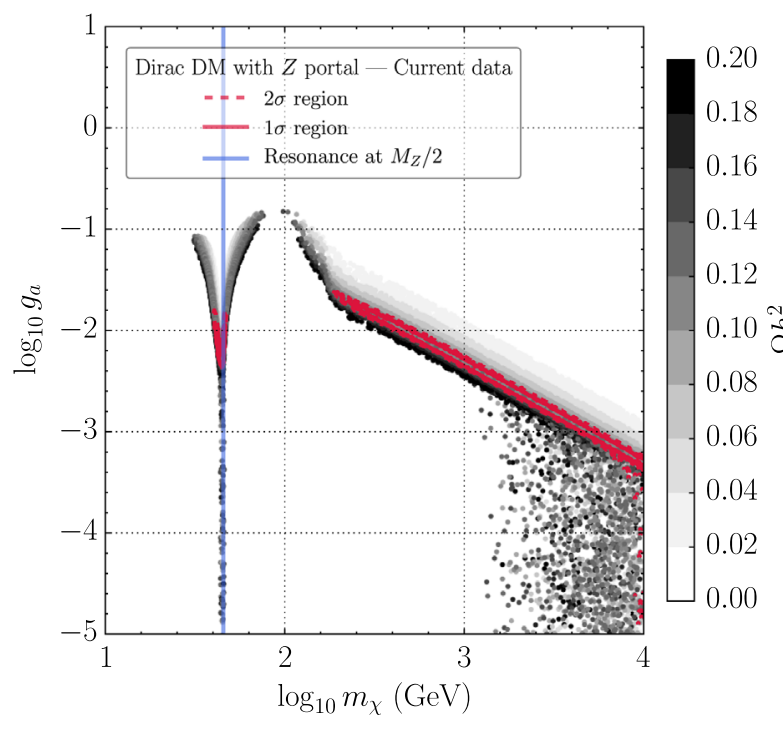

(a) Dirac DM, axial coupling

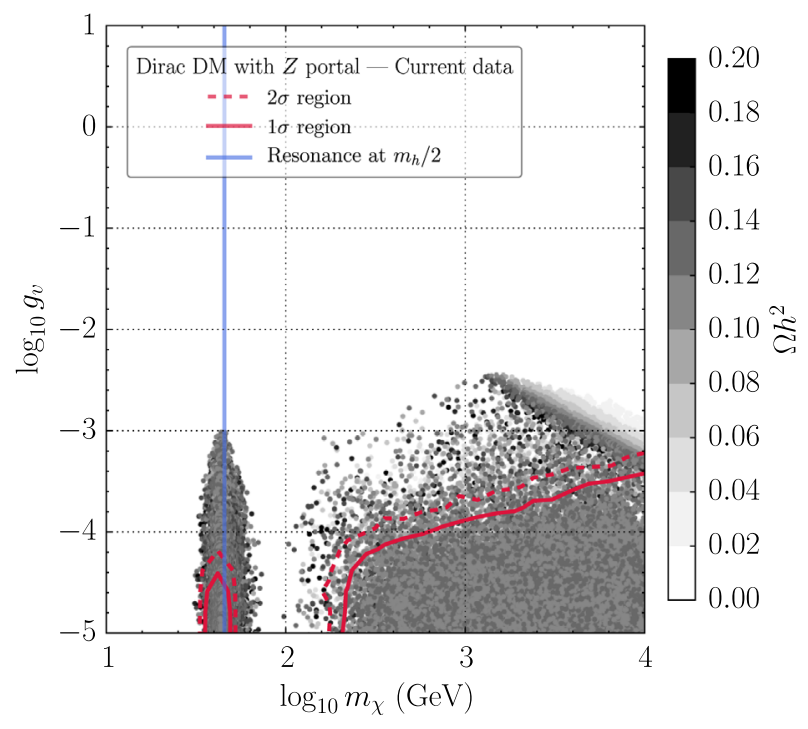

(b) Dirac DM, vector coupling

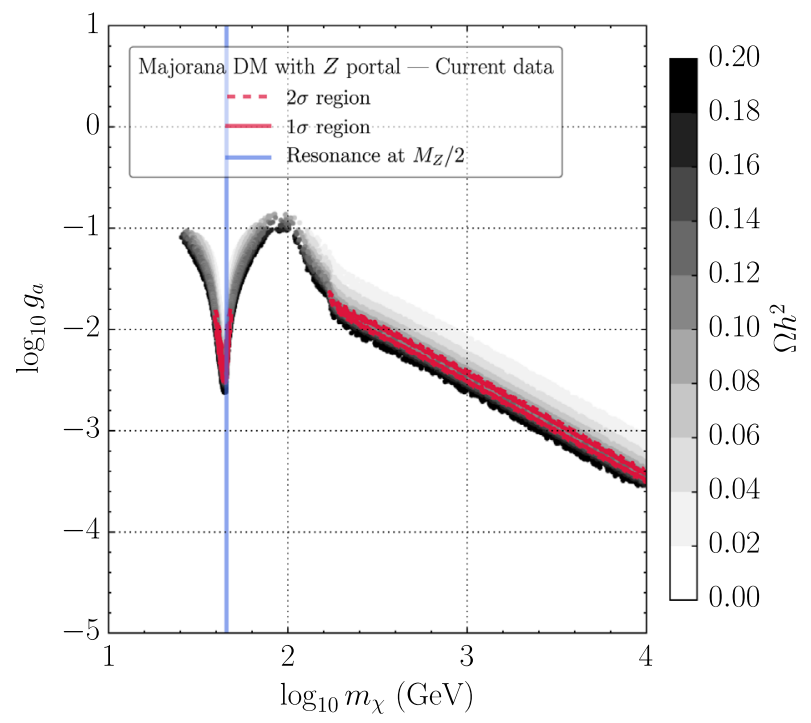

(c) Majorana DM, axial coupling

FIG. 6. Confidence regions of DM mass and coupling for Z-portal fermionic DM models in light of all current data.

points and compared multimodal and importance-nested sampling. Furthermore, we checked that we obtained similar results with an alternative technique in which we traded a coupling for the relic density, $g \rightarrow \Omega$, such that

$$
\mathcal{Z}=\left.\int \mathcal{L}(\Omega) \mathcal{L}(\cdots) \pi\left(g_{\Omega}, \cdots\right) \frac{\partial g}{\partial \Omega}\right|_{g_{\Omega}} d \Omega d \cdots
$$

We performed the resulting integral by sampling the relic density from a Gaussian likelihood, $\mathcal{L}(\Omega)$, which played the role of a prior, solving for the coupling as a function of the sampled relic density, $g_{\Omega}$, by Brent's method and calculating the derivative, which played the role of a likelihood. We found no significant changes in our evidences. To calculate confidence regions, we furthermore performed dedicated scans around the resonance to insure that it was adequately sampled.

\section{Prior sensitivity}

In our Bayesian analysis, we find that with linear priors for the DM mass and coupling the evidence is about ten times greater than that with logarithmic priors for the real scalar Higgs portal with current data and with current data except direct detection experiments. This can be ascribed to the fact that linear priors favor larger values for the DM mass, where experimental constraints from direct searches are weaker. A factor of 10 would not alter qualitative 
TABLE III. PBFs for DM models in light of DD experiments. The PBFs show the change in plausibility relative to a model unaffected by DD experiments when DD data are considered in addition to measurements of the relic abundance and collider and indirect detection data. The color scheme reflects interpretation with the Jeffreys' scale [76]: green indicates barely worth mentioning; orange indicates substantial evidence; and red indicates strong, very strong, or decisive evidence.

\begin{tabular}{lccc}
\hline \hline & & Damage to plausibility from DD \\
\cline { 2 - 4 } Model & Present data & Possible future data & Neutrino floor \\
\hline Real scalar $h$ portal & 0.3 & 0.006 & $5 \times 10^{-5}$ \\
Complex scalar $h$ portal & 0.1 & 0.002 & $1 \times 10^{-5}$ \\
Real vector $h$ portal & 0.1 & 0.0009 & $9 \times 10^{-7}$ \\
Complex vector $h$ portal & 0.02 & 0.001 & $6 \times 10^{-10}$ \\
Majorana $h$ portal & 0.2 & 0.2 & 0.1 \\
Dirac $h$ portal & 0.2 & 0.1 & 0.1 \\
Scalar $Z$ portal & $1 \times 10^{-14}$ & $7 \times 10^{-73}$ & $7 \times 10^{-129}$ \\
Vector $Z$ portal & $3 \times 10^{-10}$ & $7 \times 10^{-54}$ & $2 \times 10^{-101}$ \\
Majorana $Z$ portal & 0.3 & 0.2 & 0.1 \\
Dirac $Z$ portal & 0.08 & 0.04 & 0.01 \\
\hline \hline
\end{tabular}

TABLE IV. Change in $\chi^{2}$ for DM models in light of DD experiments in addition to measurements of the relic abundance and collider and indirect detection data. The color scheme reflects interpretation: green indicates barely worth mentioning; orange indicates substantial evidence; and red indicates strong, very strong, or decisive evidence.

\begin{tabular}{lccc}
\hline \hline & & Change in $\min \chi^{2}$ from DD data \\
\cline { 2 - 4 } Model & Present data & Possible future data & Neutrino floor \\
\hline Real scalar $h$ portal & 0 & 0 & 0.87 \\
Complex scalar $h$ portal & 0 & 0 & 2.4 \\
Real vector $h$ portal & 0 & 0 & 8.5 \\
Complex vector $h$ portal & 0 & 0 & 14 \\
Majorana $h$ portal & 0 & 0 & 0 \\
Dirac $h$ portal & 0 & 0 & 0 \\
Scalar $Z$ portal & 52 & $3.2 \times 10^{2}$ & $5.7 \times 10^{2}$ \\
Vector $Z$ portal & 33 & $2.3 \times 10^{2}$ & $4.5 \times 10^{2}$ \\
Majorana $Z$ portal & 0 & 0 & 0 \\
Dirac $Z$ portal & 0 & 0 & 0 \\
\hline \hline
\end{tabular}

conclusions drawn from our Bayes factors in Table III, as Bayes factors are typically interpreted on a logarithmic scale.

For the Majorana $Z$-boson portal, we find evidences about 100 times smaller with linear priors than with logarithmic priors with current data and also when the direct detection experiments are omitted. This arises because a small coupling, as required by this model, is disfavored by a linear prior. Factors of 100 may alter qualitative conclusions drawn from the Bayes factors in Table II. This is not surprising; with qualitatively different prior beliefs about the scales of couplings and mass of DM, one may reach qualitatively different conclusions about the relative plausibility of SM-portal models. However, our default is to present evidences from logarithmic priors, since we are agnostic about the order of magnitude of the DM mass and couplings, whereas linear priors, on the other hand, favor the greatest permitted orders of magnitude.

The partial Bayes factors in Table III (which involve ratios of the aforementioned evidences) are rather insensitive to the choice of logarithmic or linear priors, suggesting

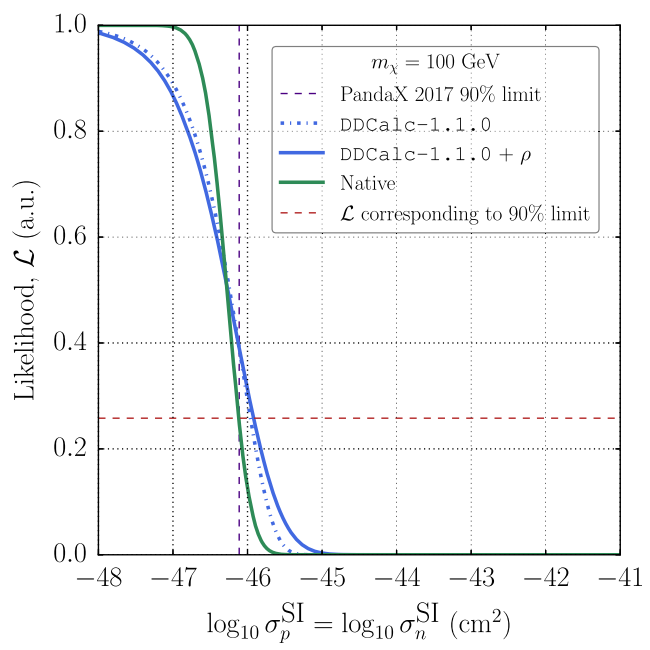

FIG. 7. Likelihood as a function of the scattering cross section for PandaX with our implementation (green) and that from DDCALC1.1.0 convolved with uncertainty in the local density (blue). The mass of the DM was fixed to $m_{\chi}=100 \mathrm{GeV}$. Note that DDCALC1.1.0 contains the fractionally weaker XENON1T 2017 data. 
TABLE V. Impact of uncertainties in evidence for real scalar Higgs-portal model with DD, ID, relic abundance, and collider data. We compare results from our native implementation of DD likelihoods and DDCALC1.1.0; only the latter allows velocity profile uncertainties (denoted Vel). Note, however, that DDCALC1.1.0 includes XENON1T data, which are fractionally weaker than PandaX for larger DM masses. We denote the uncertainty in the local density of DM by $\Delta \rho$ and our alternative treatment of hadronic uncertainties (Had) by Alt.

\begin{tabular}{lccccccc}
\hline \hline & \multicolumn{7}{c}{ Uncertainties included in DD } \\
\cline { 2 - 8 } & None & $\Delta \rho$ & Had & Alt & Had $+\Delta \rho$ & Vel & Had $+\Delta \rho+$ vel \\
\hline Native & 0.000589 & 0.000996 & 0.000484 & 0.000462 & 0.000396 & 0.000496 & 0.000145 \\
DDCALC & 0.000516 & 0.000521 & 0.000471 & 0.000393 & 0.000312 & 0.060 \\
\hline \hline
\end{tabular}

that conclusions drawn from them are somewhat robust. The frequentist $\chi^{2}$ results, which we compare with the Bayesian analysis, are of course insensitive to our choice of prior.

\section{Number of constrained parameters}

Following Refs. [99-101], we consider the effective number of constrained parameters in each model,

$$
n_{\mathrm{eff}}=\left\langle\chi^{2}\right\rangle-\min \chi^{2}
$$

where $\langle\cdot\rangle$ denotes a posterior mean. This heuristic for the number of constrained parameters originates from considering the Kullback-Leibler divergence between the prior and posterior probability density functions.

To see that this is a heuristic for the number of constrained parameters, imagine an $n$-dimensional linear model in which the likelihood is a product of Gaussians with widths $\sigma_{i}$ for each of the $n$ parameters and the priors are uniform with widths $w_{i}$. If the parameters are constrained, i.e., $\sigma_{i} \ll w_{i}$, the posterior is approximately Gaussian. Thus, $\left\langle\chi^{2}\right\rangle$ is the mean of a $\chi^{2}$ distribution with $n$ degrees of freedom, i.e., $\left\langle\chi^{2}\right\rangle=n$. The minimum $\chi^{2}=0$; thus, $n_{\mathrm{eff}}=n$.

If, on the other hand, $m$ parameters are unconstrained, i.e., $\sigma_{i} \gg w_{i}$, their posterior is approximately uniform. Thus, their contributions to $\left\langle\chi^{2}\right\rangle$ are averaged upon a uniform distribution and approximately vanish such that $\left\langle\chi^{2}\right\rangle \approx n-m$, and we find $n_{\text {eff }} \approx n-m$. This example

TABLE VI. Effective number of constrained parameters in each model for DD, ID, relic abundance, and collider data, as calculated using the prescription of Refs. [99-101].

\begin{tabular}{lc}
\hline \hline Model & $n_{\text {eff }}$ \\
\hline Real scalar $h$ portal & 1.6 \\
Complex scalar $h$ portal & 2 \\
Real vector $h$ portal & 2.6 \\
Complex vector $h$ portal & 4.1 \\
Majorana $h$ portal & 0.99 \\
Dirac $h$ portal & 0.98 \\
Scalar $Z$ portal & 3.3 \\
Vector $Z$ portal & 3.3 \\
Majorana $Z$ portal & 1.4 \\
Dirac $Z$ portal & 1.5 \\
\hline \hline
\end{tabular}

demonstrates that whether a parameter should be considered constrained depends upon our prior.

The effective number of parameters for each model is shown in Table VI. We find that the effective numbers of parameters range from about 1-3, as expected. Reference [100] argues that when evidences are similar we should discriminate between models by their numbers of constrained parameters, favoring models with fewer. While not necessarily endorsing this point of view, we note that we find that Higgs-portal models have fewer constrained parameters than Z-portal models.

\section{CONCLUSIONS}

We have presented Bayesian and frequentist appraisals of models with DM of spin $0,1 / 2$, or 1 that interacts with SM particles and annihilates via interactions with the SM Higgs or $Z$ boson, in light of constraints from Planck, DD, ID, and LHC experiments. We have also considered the possible impacts of null results from future DD searches at LZ and PICO. The Bayesian and frequentist analyses yield similar conclusions, and our results are relatively insensitive to the uncertainties in the DD scattering matrix elements and to the choice of Bayesian priors.

We find that all the Higgs-portal models studied are compatible with the available data and that they offer prospects for on-shell decays of the Higgs boson into pairs of DM particles, as well as allowing for the possibility of heavier DM particles that can be produced only via offshell Higgs bosons. In the case of $Z$-portal models, we find that the available data already provide decisive evidence against the spin- 0 and spin- 1 cases, though they do allow both the Majorana and Dirac spin-1/2 fermion options. However, in these cases, only off-shell $Z$ interactions are allowed. Null results from future DD experiments would provide substantial evidence against the scalar and vector Higgs-portal models, but the fermionic Higgs- and Z-portal models would still be viable. Null results of DD experiments down to neutrino "floor" levels would provide decisive evidence against the scalar and vector Higgsportal models and start to provide substantial evidence against the Dirac Z-portal model.

We argue that our statistical analyses and our investigation of uncertainties in DD may be the most comprehensive to date. It is for this reason that our results differ in 
emphasis from some of the previous literature. The underlying Lagrangian models we study are similar to those used in previous papers, we use a similar set of phenomenological constraints, and our implementations are also similar. However, we consider the entire parameter spaces, rather than slices, and our combination of statistical approaches enables quantitative and precise characterizations of the allowed parameter spaces for all the models we study, permitting more complete statements about the regions of parameter space in which they may survive, as well as how they could be probed in the future.

\section{ACKNOWLEDGMENTS}

The work of J. E. was supported in part by STFC (United Kingdom) via research Grant No. ST/L000326/1 and in part by the Estonian Research Council via Mobilitas Plus Grant No. MOBTT5. The work of L. M. and M. R. was supported by Estonian Research Council Grant No. IUT236 and by ERDF Centre of Excellence Project No. TK133.

\section{APPENDIX: STATISTICS}

We analyze SM-portal models with Bayesian and frequentist statistics. Within each framework, we require two distinct methods: a method to estimate the parameters in a DM model, e.g., the DM mass, and a method to judge the viability of the DM model. An ingredient in all calculations is the likelihood - the probability of obtaining experimental data $D$ given a parameter point $x$, in a model $M$, i.e.,

$$
\mathcal{L}(x) \equiv p(D \mid x, M)
$$

The likelihood in our analysis is a product of statistically independent likelihoods from measurements of the DM abundance and DD, ID, and LHC experiments,

$$
\mathcal{L}(x)=\mathcal{L}_{\text {Planck }}(x) \times \mathcal{L}_{\mathrm{ID}}(x) \times \mathcal{L}_{\mathrm{DD}}(x) \times \mathcal{L}_{\mathrm{LHC}}(x) .
$$

The individual likelihoods are described in Sec. III.

For parameter inference with frequentist statistics, we calculate confidence regions by Wilks's theorem. For a two-dimensional confidence region for parameters $a$ and $b$ in a model with parameters $x=\{a, b, c\}$, by Wilks's theorem,

$$
\Delta \chi^{2}(a, b) \equiv-2 \ln \frac{\mathcal{L}(a, b, \hat{c})}{\mathcal{L}(\hat{x})} \sim \chi_{2}^{2},
$$

where $\hat{x}$ are the best-fitting parameters and $\hat{c}$ is the best-fit $c$ for a particular $a$ and $b$. The, e.g., 95\% confidence interval is the region in $(a, b)$ in which $\Delta \chi^{2}(a, b) \leq 5.99$. For judging model viability with frequentist statistics, we construct a test statistic:

$$
\operatorname{minimum} \chi^{2} \equiv-2 \ln \frac{\mathcal{L}(\hat{x})}{\mathcal{L}_{\max }}
$$

Unfortunately, as the distribution of the above quantity is unknown, it is not possible to calculate precisely a $p$-value, i.e., the probability of obtaining a test statistic so extreme were the null hypothesis true. See, e.g., the discussion of the difficulties in estimating the distribution in a similar analysis in Ref. [21]. For illustration, though, we calculate $p$-values assuming a $\chi^{2}$ distribution with 2 degrees of freedom, minimum $\chi^{2} \sim \chi_{2}^{2}$, which may be reasonable given the constraints and parameters in our models, and the predictions of our DM models at their best-fit points.

Our Bayesian methodology requires a further ingredient: priors, $p(x \mid M)$, for the parameters $x$ in a model $M$. The priors for our SM-portal models are discussed in Sec. II. We update the priors with experimental data by Bayes's theorem, resulting in posterior distributions

$$
p(x \mid D, M)=\frac{\mathcal{L}(x) \cdot p(x \mid M)}{p(D \mid M)} .
$$

For parameter inference, we marginalize parameters that are not of interest, e.g.,

$$
p(a, b \mid D, M)=\int p(a, b, c \mid D, M) d c,
$$

and find so-called credible regions: regions of $(a, b)$ that contain a particular fraction e.g., $95 \%$, of the posterior. The regions are not unique; we use the ordering rules detailed in Ref. [98]. For judging the viability of a model, we calculate directly its change in relative plausibility [76]. This involves calculating evidence integrals,

$$
p(D \mid M)=\int d x \mathcal{L}(x) p(x \mid M) .
$$

The change in relative plausibility of models $M_{a}$ and $M_{b}$ in light of data $D$ may be found by Bayes's theorem,

$$
\frac{\frac{p\left(M_{a} \mid D\right)}{p\left(M_{b} \mid D\right)}}{\frac{p\left(M_{a}\right)}{p\left(M_{b}\right)}}=\frac{p\left(D \mid M_{a}\right)}{p\left(D \mid M_{b}\right)} .
$$

This may be expressed in words as

$$
\begin{aligned}
& \text { change in relative plausibility } \\
& \qquad=\frac{\text { posterior odds }}{\text { prior odds }}=\text { Bayes factor }
\end{aligned}
$$

Thus, we calculate Bayes factors, which are ratios of evidences, to judge changes in the relative plausibility of SM-portal models in light of data. 
TABLE VII. Experimental data and projected data in our scans. The symbol $\otimes$ denotes a convolution of a limit with an uncertainty; e.g., $\otimes \Delta \rho_{\text {DM }}$ denotes convolution with uncertainty in the local density of DM.

Relic density

$\Omega h^{2}$

Invisible widths

$\Gamma_{Z}^{\text {inv }}$

$\mathrm{BR}_{h}^{\mathrm{inv}}$

$0.1199 \pm 0.0022 \pm 10 \%$

Gaussian

Gaussian

Likelhood was published

$\lesssim 0.24$

Direct detection

$\begin{aligned} \sigma_{\mathrm{SI}}^{p, n} & \lesssim 10^{-46} \mathrm{~cm}^{2} \\ \sigma_{\mathrm{SD}}^{p} & \lesssim 10^{-40} \mathrm{~cm}^{2} \\ \sigma_{\mathrm{SD}}^{n} & \lesssim 10^{-40} \mathrm{~cm}^{2}\end{aligned}$

Projected direct detection

$\begin{aligned} \sigma_{\mathrm{SI}}^{p, n} & \lesssim 10^{-47} \mathrm{~cm}^{2} \\ \sigma_{\mathrm{SD}}^{p} & \lesssim 10^{-42} \mathrm{~cm}^{2} \\ \sigma_{\mathrm{SD}}^{n} & \lesssim 10^{-42} \mathrm{~cm}^{2}\end{aligned}$

Spin-independent direct detection floor from coherent neutrino scattering backgrounds

$\begin{array}{lll}\sigma_{\mathrm{SI}}^{p, n} & \lesssim 10^{-49} \mathrm{~cm}^{2} & \text { Discovery limit } \otimes \Delta \rho_{\mathrm{DM}} \\ \sigma_{\mathrm{SD}}^{p} & \lesssim 10^{-43} \mathrm{~cm}^{2} & \text { Discovery limit } \otimes \Delta \rho_{\mathrm{DM}} \\ \sigma_{\mathrm{SD}}^{n} & \lesssim 10^{-43} \mathrm{~cm}^{2} & \text { Discovery limit } \otimes \Delta \rho_{\mathrm{DM}}\end{array}$

Indirect detection- $-u \bar{u}, b \bar{b}, W W, e \bar{e}, \mu \bar{\mu}$, and $\tau \bar{\tau}$ channels

$\langle\sigma v\rangle \quad \lesssim 10^{-26} \mathrm{~cm}^{3} / \mathrm{s} \quad 95 \%$ limit $\otimes J$-factors

LHC_-see Table I
$90 \%$ limit $\otimes \Delta \rho_{\mathrm{DM}}$

$90 \%$ limit $\otimes \Delta \rho_{\mathrm{DM}}$

$90 \%$ limit $\otimes \Delta \rho_{\mathrm{DM}}$

$90 \%$ limit $\otimes \Delta \rho_{\mathrm{DM}}$

$90 \%$ limit $\otimes \Delta \rho_{\mathrm{DM}}$

$90 \%$ limit $\otimes \Delta \rho_{\mathrm{DM}}$
Planck [1]

LEP, PDG combination [66]

CMS [68]

PandaX [4]

PICO-60 [2]

PandaX [78]

XENONnT projection [28]

PICO-500 projection [92]

LZ projection [91]

Xe, Ruppin et al. [93]

$\mathrm{C}_{3} F_{8}$, Ruppin et al. [93]

Xe, Ruppin et al. [93]

Fermi-LAT dSphs six year [88]

TABLE VIII. Priors for model and nuisance parameters in our scans. For details of the nuclear matrix elements, see Sec. III C.

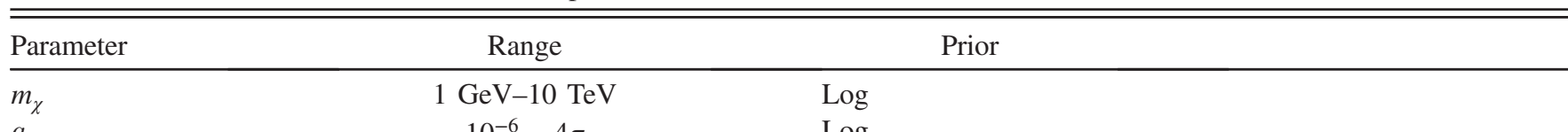

$g$

Nuclear nuisance

$\sigma_{s}$

$\sigma_{\pi N}$

$m_{u} / m_{d}$

$m_{s} / m_{d}$

$\Delta u+\Delta \bar{u}$

$\Delta d+\Delta \bar{d}$

$\Delta s+\Delta \bar{s}$

$\delta u$

$\delta d$

$\delta s$

Astrophysical nuisance

$\rho_{\text {DM }}$

$J$-factor for dSphs

\section{$0.3 \mathrm{GeV} / \mathrm{cm}^{3}$}

Log-normal, $\sigma_{\log _{10} J}=0.25[88]$ $\log$

Lattice, ETM [85]

Lattice, ETM [84] $\}$

Pheno [83]

Lattice, PDG comb. [66]

Lattice, PDG comb. [66]

HERMES [102]

HERMES [102]

HERMES [102]

Lattice [103]

Lattice [103]

Lattice [103]

Log-normal, $\sigma_{\ln \rho}=\ln 2$

Standard halo-see text; included in single scan as cross-check (distributions as in Ref. [104])

$\begin{array}{lll}v_{\text {esc }} & 550 \pm 35 \mathrm{~km} / \mathrm{s} & \text { Gaussian } \\ v_{\text {rel }} & 235 \pm 20 \mathrm{~km} / \mathrm{s} & \text { Gaussian } \\ v_{0} & 235 \pm 20 \mathrm{~km} / \mathrm{s} & \text { Gaussian }\end{array}$

SM nuisance

$M_{Z}$

$91.1876 \pm 0.0021 \mathrm{GeV}$

Gaussian $125.09 \pm 0.24 \mathrm{GeV}$

Gaussian

LHC, PDG combination [66] LEP, PDG combination [66] 
A merit of the Bayes factor, which is particularly relevant to our analysis of DM models, is that we may judge damage to the plausibility of a model caused by experiments that disfavor a model without excluding its entire parameter space. With $p$-values, the status of a DM model would change only once the entirety of its parameter space was excluded by, e.g., DD experiments. With Bayes factors, however, the plausibility of a DM model would deteriorate in light of failed searches, even if there remained parameter points that are not excluded.
[1] P. A. R. Ade et al., Planck 2015 results. XIII. Cosmological parameters, Astron. Astrophys. 594, A13 (2016).

[2] C. Amole et al., Dark Matter Search Results from the PICO-60 $\mathrm{C}_{3} \mathrm{~F}_{8}$ Bubble Chamber, Phys. Rev. Lett. 118, 251301 (2017).

[3] E. Aprile et al., First Dark Matter Search Results from the XENON1T Experiment, Phys. Rev. Lett. 119, 181301 (2017).

[4] X. Cui et al., Dark Matter Results From 54-Ton-Day Exposure of PandaX-II Experiment, Phys. Rev. Lett. 119, 181302 (2017).

[5] V. Khachatryan et al., Search for dark matter, extra dimensions, and unparticles in monojet events in proton-proton collisions at $\sqrt{s}=8 \mathrm{TeV}$, Eur. Phys. J. C 75, 235 (2015).

[6] G. Aad et al., Search for pair-produced third-generation squarks decaying via charm quarks or in compressed supersymmetric scenarios in $p p$ collisions at $\sqrt{s}=$ $8 \mathrm{TeV}$ with the ATLAS detector, Phys. Rev. D 90, 052008 (2014).

[7] G. Aad et al., Search for new phenomena in final states with an energetic jet and large missing transverse momentum in pp collisions at $\sqrt{s}=8 \mathrm{TeV}$ with the ATLAS detector, Eur. Phys. J. C 75, 299 (2015); Erratum, 75, 408 (2015).

[8] M. Aaboud et al., Search for new phenomena in final states with an energetic jet and large missing transverse momentum in $p p$ collisions at $\sqrt{s}=13 \mathrm{TeV}$ using the ATLAS detector, Phys. Rev. D 94, 032005 (2016).

[9] M. Aaboud et al., Search for dark matter at $\sqrt{s}=13 \mathrm{TeV}$ in final states containing an energetic photon and large missing transverse momentum with the ATLAS detector, Eur. Phys. J. C 77, 393 (2017).

[10] G. Aad et al., Search for new phenomena in events with a photon and missing transverse momentum in $p p$ collisions at $\sqrt{s}=8 \mathrm{TeV}$ with the ATLAS detector, Phys. Rev. D 91, 012008 (2015); Erratum, 92, 059903 (2015).

[11] M. Aaboud et al., Search for new phenomena in events with a photon and missing transverse momentum in $p p$ collisions at $\sqrt{s}=13 \mathrm{TeV}$ with the ATLAS detector, J. High Energy Phys. 06 (2016) 059.

[12] L. E. Ibanez and G. G. Ross, Discrete gauge symmetries and the origin of baryon and lepton number conservation in supersymmetric versions of the standard model, Nucl. Phys. B368, 3 (1992).

[13] J. McDonald, Gauge singlet scalars as cold dark matter, Phys. Rev. D 50, 3637 (1994).
[14] G. Belanger, K. Kannike, A. Pukhov, and M. Raidal, $Z_{3}$ scalar singlet dark matter, J. Cosmol. Astropart. Phys. 01 (2013) 022.

[15] H. K. Dreiner, C. Luhn, and M. Thormeier, What is the discrete gauge symmetry of the MSSM?, Phys. Rev. D 73, 075007 (2006).

[16] G. Bélanger, K. Kannike, A. Pukhov, and M. Raidal, Minimal semi-annihilating $\mathbb{Z}_{N}$ scalar dark matter, J. Cosmol. Astropart. Phys. 06 (2014) 021.

[17] M. Escudero, A. Berlin, D. Hooper, and M.-X. Lin, Toward (finally!) ruling out $Z$ and Higgs mediated dark matter models, J. Cosmol. Astropart. Phys. 12 (2016) 029.

[18] G. Arcadi, M. Dutra, P. Ghosh, M. Lindner, Y. Mambrini, M. Pierre, S. Profumo, and F. S. Queiroz, The waning of the WIMP? A review of models, searches, and constraints, Eur. Phys. J. C 78, 203 (2018).

[19] A. Cuoco, B. Eiteneuer, J. Heisig, and M. Krämer, A global fit of the $\gamma$-ray galactic center excess within the scalar singlet Higgs portal model, J. Cosmol. Astropart. Phys. 06 (2016) 050.

[20] X.-G. He and J. Tandean, New LUX and PandaX-II results illuminating the simplest Higgs-portal dark matter models, J. High Energy Phys. 12 (2016) 074.

[21] P. Athron et al., Status of the scalar singlet dark matter model, Eur. Phys. J. C 77, 568 (2017).

[22] N. F. Bell, G. Busoni, and I. W. Sanderson, Two Higgs doublet dark matter portal, J. Cosmol. Astropart. Phys. 01 (2018) 015.

[23] A. Beniwal, F. Rajec, C. Savage, P. Scott, C. Weniger, M. White, and A. G. Williams, Combined analysis of effective Higgs portal dark matter models, Phys. Rev. D 93, 115016 (2016).

[24] C.-F. Chang, X.-G. He, and J. Tandean, Two-Higgsdoublet-portal dark-matter models in light of direct search and LHC data, J. High Energy Phys. 04 (2017) 107.

[25] S. Baum, R. Catena, J. Conrad, K. Freese, and M. B. Krauss, Determining dark matter properties with a XENONnT/LZ signal and LHC-Run3 mono-jet searches, Phys. Rev. D 97, 083002 (2018).

[26] J. A. Casas, D. G. Cerdeño, J. M. Moreno, and J. Quilis, Reopening the Higgs portal for single scalar dark matter, J. High Energy Phys. 05 (2017) 036.

[27] B. J. Mount et al., LUX-ZEPLIN (LZ) Technical Design Report, 2017.

[28] E. Aprile et al., Physics reach of the XENON1T dark matter experiment, J. Cosmol. Astropart. Phys. 04 (2016) 027. 
[29] A. Berlin, D. Hooper, and S. D. McDermott, Simplified dark matter models for the galactic center gamma-ray excess, Phys. Rev. D 89, 115022 (2014).

[30] M. R. Buckley, D. Feld, and D. Goncalves, Scalar simplified models for dark matter, Phys. Rev. D 91, 015017 (2015).

[31] O. Buchmueller, M. J. Dolan, S. A. Malik, and C. McCabe, Characterising dark matter searches at colliders and direct detection experiments: Vector mediators, J. High Energy Phys. 01 (2015) 037.

[32] A. DiFranzo, K. I. Nagao, A. Rajaraman, and T. M. P. Tait, Simplified models for dark matter interacting with quarks, J. High Energy Phys. 11 (2013) 014; Erratum, 01 (2014) 162.

[33] P. Harris, V. V. Khoze, M. Spannowsky, and C. Williams, Constraining dark sectors at colliders: Beyond the effective theory approach, Phys. Rev. D 91, 055009 (2015).

[34] D. Abercrombie et al., Dark matter benchmark models for early LHC run-2searches: Report of the ATLAS/CMS Dark Matter Forum, arXiv:1507.00966.

[35] J. Abdallah et al., Simplified models for dark matter searches at the LHC, Phys. Dark Universe 9, 8 (2015).

[36] D. Alves, Simplified models for LHC new physics searches, J. Phys. G 39, 105005 (2012).

[37] G. Busoni et al., Recommendations on presenting LHC searches for missing transverse energy signals using simplified $s$-channel models of dark matter, arXiv:1603.04156.

[38] S. Kraml, S. Kulkarni, U. Laa, A. Lessa, W. Magerl, D. Proschofsky-Spindler, and W. Waltenberger, SModelS: A tool for interpreting simplified-model results from the LHC and its application to supersymmetry, Eur. Phys. J. C 74, 2868 (2014).

[39] A. Choudhury, K. Kowalska, L. Roszkowski, E. M. Sessolo, and A. J. Williams, Less-simplified models of dark matter for direct detection and the LHC, J. High Energy Phys. 04 (2016) 182.

[40] S. Chatrchyan et al., Interpretation of searches for supersymmetry with simplified models, Phys. Rev. D 88, 052017 (2013).

[41] T. Cohen, T. Golling, M. Hance, A. Henrichs, K. Howe, J. Loyal, S. Padhi, and J.G. Wacker, SUSY simplified models at 14, 33, and $100 \mathrm{TeV}$ proton colliders, J. High Energy Phys. 04 (2014) 117.

[42] N. D. Christensen and C. Duhr, FeynRules-Feynman rules made easy, Comput. Phys. Commun. 180, 1614 (2009).

[43] N. D. Christensen, P. de Aquino, C. Degrande, C. Duhr, B. Fuks, M. Herquet, F. Maltoni, and S. Schumann, A comprehensive approach to new physics simulations, Eur. Phys. J. C 71, 1541 (2011).

[44] G. Belanger, F. Boudjema, A. Pukhov, and A. Semenov, MicrOMEGAs: A program for calculating the relic density in the MSSM, Comput. Phys. Commun. 149, 103 (2002).

[45] D. Barducci, G. Belanger, J. Bernon, F. Boudjema, J. Da Silva, S. Kraml, U. Laa, and A. Pukhov, Collider limits on new physics within micrOMEGAs_4.3, Comput. Phys. Commun. 222, 327 (2018).

[46] A. Belyaev, N. D. Christensen, and A. Pukhov, CalcHEP 3.4 for collider physics within and beyond the Standard Model, Comput. Phys. Commun. 184, 1729 (2013).

[47] Y. G. Kim, K. Y. Lee, and S. Shin, Singlet fermionic dark matter, J. High Energy Phys. 05 (2008) 100.
[48] C. Englert, T. Plehn, D. Zerwas, and P. M. Zerwas, Exploring the Higgs portal, Phys. Lett. B 703, 298 (2011).

[49] C. P. Burgess, M. Pospelov, and T. ter Veldhuis, The minimal model of nonbaryonic dark matter: A singlet scalar, Nucl. Phys. B619, 709 (2001).

[50] G. Cynolter, E. Lendvai, and G. Pocsik, Note on unitarity constraints in a model for a singlet scalar dark matter candidate, Acta Phys. Pol. B 36, 827 (2005).

[51] H. Ruegg and M. Ruiz-Altaba, The Stueckelberg field, Int. J. Mod. Phys. A 19, 3265 (2004).

[52] A. DiFranzo, P. J. Fox, and T. M. P. Tait, Vector dark matter through a radiative Higgs portal, J. High Energy Phys. 04 (2016) 135.

[53] M. Duch, B. Grzadkowski, and M. McGarrie, A stable Higgs portal with vector dark matter, J. High Energy Phys. 09 (2015) 162.

[54] O. Lebedev, H. M. Lee, and Y. Mambrini, Vector Higgsportal dark matter and the invisible Higgs, Phys. Lett. B 707, 570 (2012).

[55] T. Hambye, Hidden vector dark matter, J. High Energy Phys. 01 (2009) 028.

[56] J. Kearney, N. Orlofsky, and A. Pierce, $Z$ boson mediated dark matter beyond the effective theory, Phys. Rev. D 95 , 035020 (2017).

[57] A. De Simone, G. Francesco Giudice, and A. Strumia, Benchmarks for dark matter searches at the LHC, J. High Energy Phys. 06 (2014) 081.

[58] G. Arcadi, Y. Mambrini, and F. Richard, Z-portal dark matter, J. Cosmol. Astropart. Phys. 03 (2015) 018.

[59] N. Nagata and S. Shirai, Electroweakly-interacting Dirac dark matter, Phys. Rev. D 91, 055035 (2015).

[60] P. Anastasopoulos, M. Bianchi, E. Dudas, and E. Kiritsis, Anomalies, anomalous U(1)'s and generalized ChernSimons terms, J. High Energy Phys. 11 (2006) 057.

[61] I. Antoniadis, A. Boyarsky, S. Espahbodi, O. Ruchayskiy, and J.D. Wells, Anomaly driven signatures of new invisible physics at the Large Hadron Collider, Nucl. Phys. B824, 296 (2010).

[62] E. Dudas, Y. Mambrini, S. Pokorski, and A. Romagnoni, (In)visible Z-prime and dark matter, J. High Energy Phys. 08 (2009) 014.

[63] E. Dudas, L. Heurtier, Y. Mambrini, and B. Zaldivar, Extra $\mathrm{U}(1)$, effective operators, anomalies and dark matter, J. High Energy Phys. 11 (2013) 083.

[64] T. Binder, T. Bringmann, M. Gustafsson, and A. Hryczuk, Early kinetic decoupling of dark matter: When the standard way of calculating the thermal relic density fails, Phys. Rev. D 96, 115010 (2017).

[65] M. Duch and B. Grzadkowski, Resonance enhancement of dark matter interactions: The case for early kinetic decoupling and velocity dependent resonance width, J. High Energy Phys. 09 (2017) 159.

[66] C. Patrignani et al., Review of particle physics, Chin. Phys. C 40, 100001 (2016).

[67] A. Freitas, Higher-order electroweak corrections to the partial widths and branching ratios of the $\mathrm{Z}$ boson, J. High Energy Phys. 04 (2014) 070.

[68] V. Khachatryan et al. (The CMS collaboration), Searches for invisible decays of the Higgs boson in pp collisions at 
$\sqrt{s}=7,8$, and $13 \mathrm{TeV}$, J. High Energy Phys. 02 (2014) 057.

[69] D. Dercks, N. Desai, J. S. Kim, K. Rolbiecki, J. Tattersall, and T. Weber, CheckMATE 2: From the model to the limit, Comput. Phys. Commun. 221, 383 (2017).

[70] J. de Favereau, C. Delaere, P. Demin, A. Giammanco, V. Lemaître, A. Mertens, and M. Selvaggi, DELPHES 3, A modular framework for fast simulation of a generic collider experiment, J. High Energy Phys. 02 (2014) 057.

[71] M. Cacciari, G. P. Salam, and G. Soyez, FastJet user manual, Eur. Phys. J. C 72, 1896 (2012).

[72] M. Cacciari and G. P. Salam, Dispelling the $N^{3}$ myth for the $k_{t}$ jet-finder, Phys. Lett. B 641, 57 (2006).

[73] A. L. Read, Presentation of search results: The $\mathrm{CL}_{s}$ technique, J. Phys. G 28, 2693 (2002).

[74] M. Cacciari, G. P. Salam, and G. Soyez, The anti- $k_{t}$ jet clustering algorithm, J. High Energy Phys. 04 (2008) 063.

[75] M. Aaboud et al., Search for dark matter and other new phenomena in events with an energetic jet and large missing transverse momentum using the ATLAS detector, J. High Energy Phys. 01 (2018) 126.

[76] H. Jeffreys, The Theory of Probability, Oxford Classic Texts in the Physical Sciences (Oxford University, New York, 1939).

[77] J. Alwall, R. Frederix, S. Frixione, V. Hirschi, F. Maltoni, O. Mattelaer, H. S. Shao, T. Stelzer, P. Torrielli, and M. Zaro, The automated computation of tree-level and nextto-leading order differential cross sections, and their matching to parton shower simulations, J. High Energy Phys. 07 (2014) 079.

[78] C. Fu et al., Spin-Dependent Weakly-Interacting-MassiveParticle-Nucleon Cross Section Limits from First Data of PandaX-II Experiment, Phys. Rev. Lett. 118, 071301 (2017).

[79] G. Belanger, F. Boudjema, A. Pukhov, and A. Semenov, Dark matter direct detection rate in a generic model with micrOMEGAs 2.2, Comput. Phys. Commun. 180, 747 (2009).

[80] P. E. Shanahan, Chiral effective theory methods and their application to the structure of hadrons from lattice QCD, J. Phys. G 43, 124001 (2016).

[81] J. M. Alarcon, J. Martin Camalich, and J. A. Oller, The chiral representation of the $\pi N$ scattering amplitude and the pion-nucleon sigma term, Phys. Rev. D 85, 051503 (2012).

[82] J. M. Alarcon, L. S. Geng, J. M. Camalich, and J. A. Oller, The strangeness content of the nucleon from effective field theory and phenomenology, Phys. Lett. B 730, 342 (2014).

[83] M. Hoferichter, J. Ruiz de Elvira, B. Kubis, and U.-G. Meißner, High-Precision Determination of the PionNucleon $\sigma$ Term from Roy-Steiner Equations, Phys. Rev. Lett. 115, 092301 (2015).

[84] J. Ruiz de Elvira, M. Hoferichter, B. Kubis, and U.-G. Meißner, Extracting the sigma-term from low-energy pion-nucleon scattering, J. Phys. G 45, 024001 (2018).

[85] A. Abdel-Rehim, C. Alexandrou, M. Constantinou, K. Hadjiyiannakou, K. Jansen, Ch. Kallidonis, G. Koutsou, and A. V. Aviles-Casco, Direct Evaluation of the Quark Content of Nucleons from Lattice QCD at the Physical Point, Phys. Rev. Lett. 116, 252001 (2016).
[86] M. Hoferichter, P. Klos, J. Menéndez, and A. Schwenk, Improved Limits for Higgs-Portal Dark Matter from LHC Searches, Phys. Rev. Lett. 119, 181803 (2017).

[87] T. Bringmann et al., DDCalc: Dark matter direct detection phenomenology package, http://ddcalc.hepforge.org/.

[88] M. Ackermann et al., Searching for Dark Matter Annihilation from Milky Way Dwarf Spheroidal Galaxies with Six Years of Fermi Large Area Telescope Data, Phys. Rev. Lett. 115, 231301 (2015).

[89] M. G. Aartsen et al., Search for annihilating dark matter in the Sun with 3 years of IceCube data, Eur. Phys. J. C 77, 146 (2017).

[90] E. Bagnaschi et al., Likelihood analysis of the pMSSM11 in light of LHC 13-TeV data, Eur. Phys. J. C 78, 256 (2018).

[91] P. Cushman et al., Working Group report: WIMP dark matter direct detection, arXiv:1310.8327.

[92] C. Krauss, PICO dark matter searches, ICHEP, 2016, https: //indico.cern.ch/event/432527/contributions/1071434/.

[93] F. Ruppin, J. Billard, E. Figueroa-Feliciano, and L. Strigari, Complementarity of dark matter detectors in light of the neutrino background, Phys. Rev. D 90, 083510 (2014).

[94] J. Buchner, A. Georgakakis, K. Nandra, L. Hsu, C. Rangel, M. Brightman, A. Merloni, M. Salvato, J. Donley, and D. Kocevski, X-ray spectral modelling of the AGN obscuring region in the CDFS: Bayesian model selection and catalogue, Astron. Astrophys. 564, A125 (2014).

[95] F. Feroz and M. P. Hobson, Multimodal nested sampling: An efficient and robust alternative to MCMC methods for astronomical data analysis, Mon. Not. R. Astron. Soc. 384, 449 (2008).

[96] F. Feroz, M. P. Hobson, and M. Bridges, MultiNest: An efficient and robust Bayesian inference tool for cosmology and particle physics, Mon. Not. R. Astron. Soc. 398, 1601 (2009).

[97] F. Feroz, M. P. Hobson, E. Cameron, and A. N. Pettitt, Importance nested sampling and the MultiNest algorithm, arXiv:1306.2144.

[98] A. Fowlie and M. H. Bardsley, Superplot: A graphical interface for plotting and analysing MultiNest output, Eur. Phys. J. Plus 131, 391 (2016).

[99] D. J. Spiegelhalter, N. G. Best, B. P. Carlin, and A. Van Der Linde, Bayesian measures of model complexity and fit, J. R. Stat. Soc., Ser. B 64, 583 (2002).

[100] M. Kunz, R. Trotta, and D. Parkinson, Measuring the effective complexity of cosmological models, Phys. Rev. D 74, 023503 (2006).

[101] J. Martin, C. Ringeval, R. Trotta, and V. Vennin, The best inflationary models after Planck, J. Cosmol. Astropart. Phys. 03 (2014) 039.

[102] A. Airapetian et al., Precise determination of the spin structure function $\mathrm{g}(1)$ of the proton, deuteron and neutron, Phys. Rev. D 75, 012007 (2007).

[103] S. Aoki, M. Doui, T. Hatsuda, and Y. Kuramashi, Tensor charge of the nucleon in lattice QCD, Phys. Rev. D 56, 433 (1997).

[104] T. Bringmann et al., DarkBit: A GAMBIT module for computing dark matter observables and likelihoods, Eur. Phys. J. C 77, 831 (2017). 